\title{
A Bound on the Total Chromatic Number
}

\author{
Michael Molloy* \\ Department of Computer Science \\ University of Toronto \\ Toronto, Canada \\ Bruce Reed* \\ Equipe Combinatoire \\ CNRS \\ Université Pierre et Marie Curie \\ Paris, France
}

November 2, 2000

\begin{abstract}
We prove that the total chromatic number of any graph with maximum degree $\Delta$ is at most $\Delta$ plus an absolute constant. In particular, we show that for $\Delta$ sufficiently large, the total chromatic number of such a graph is at most $\Delta+10^{26}$. The proof is probabilistic.
\end{abstract}

\section{Introduction}

A total colouring of a graph $G$ is an assignment of colours to its vertices and edges so that no two adjacent vertices have the same colour, no two adjacent edges have the same colour, and no edge has the same colour as one of its

*This research was supported by NATO Collaborative Research Grant \#CRG950235. 
endpoints. The total chromatic number, $\chi^{\prime \prime}(G)$ is the least number of colours required for a total colouring of $G$.

This concept was introduced independently by Behzad [5] and Vizing [22], who each conjectured that any simple graph with maximum degree $\Delta$ has a $\Delta+2$ total colouring. Note that if true, this conjecture is tight as every such graph requires at least $\Delta+1$ colours and there are some graphs such as $K_{\Delta+1}, \Delta$ odd, which require $\Delta+2$ colours. Kilakos and Reed [15] have shown that the fractional total chromatic number is at most $\Delta+2$. For more information on total colouring, see the recent book by Yap [23].

The first $\Delta+o(\Delta)$ bound on the total chromatic number of such a graph was $\Delta+2 \sqrt{\Delta}$, due to Hind [11]. More recently, Häggkvist and Chetwynd[10] have improved this bound to $\Delta+18 \Delta^{1 / 3} \log (3 \Delta)$. In [13], Hind, Molloy and Reed improve this bound further to $\Delta+\operatorname{poly}(\log \Delta)$. Brualdi [6] and Alon [1] asked whether the total chromatic number of such graphs is at most $\Delta+C$ for some absolute constant $C$. Here, we answer this question positively:

Theorem 1.1 If a simple graph $G$ has maximum degree $\Delta$ at least as large as a particular constant, then $\chi^{\prime \prime}(G) \leq \Delta+C$, where $C=10^{26}$.

Remark 1.2 We do not attempt to optimize our constant $C$, prefering rather to choose a value which provides a simpler presentation. In fact, by adding a few more intricacies to our proof, and being a little more careful in our calculations, we can obtain $C=500$, and this technique will probably yield $C$ near 100 . However, it does not appear that this technique will yield a value of $C$ which is very close to 2 , say less than 10 .

We make no attempt to find the neccessary lower bound on $\Delta$, only insisting that $\Delta$ satisfies various implicit conditions. Note that, using for example the aforementioned result of Hind, Theorem 1 implies the existence of a constant $C^{\prime}$, such that for any $\Delta$ and any $G$ with maximum degree $\Delta$, $\chi^{\prime \prime}(G) \leq \Delta+C^{\prime}$.

Our proof relies heavily on several applications of the Lovász Local Lemma. If $\Delta$ does not grow too quickly with $n$, say $\Delta=o\left(\log ^{1 / 3} n\right.$ ) (for example if $\Delta$ is fixed) then the technique of Beck[4] can be applied to make the argument constructive, providing a polytime algorithm to find a $\Delta+C$ total colouring of our graph. See [19] or [20] for details.

In [13], we show that if we begin with any $\Delta+1$ vertex colouring of $G$, satisfying a particular condition (where the existance of such a colouring is 
guaranteed in [12]), then we can always complete this colouring to a total colouring of $G$ using at most $\Delta+$ poly $(\log \Delta)$ colours. Here, we take the opposite approach. We show that if we begin with any $\Delta+1$ edge colouring of $G$, then we can essentially complete it to a total colouring of $G$ using at most $\Delta+C$ colours. By "essentially", we mean this: There will be a subgraph, $R \subseteq G$ called a reject graph, such that upon deleting the colours on the edges of $R$, we have a partial total colouring of $G$, using $\Delta+1$ colours, where only the edges of $R$ are uncoloured. Furthermore, we will guarantee that $R$ has maximum degree at most $C-2$, and so by Vizing's Theorem, we can edge-colour $R$ using $C-1$ new colours, thus obtaining our $\Delta+C$ total colouring.

In [18], we show that for any simple graph $G$ with maximum degree $\Delta$, if the neighbourhood of each vertex of $G$ has at most $(1-\epsilon)\left(\begin{array}{l}\Delta \\ 2\end{array}\right)$ edges for some $\epsilon>0$, then $\chi(G) \leq(1-\delta) \Delta$, for some $\delta=\delta(\epsilon)>0$. Reed [21] extends this result to show that if the size of the largest clique in $G$ is at most $(1-\epsilon) \Delta$ then $\chi(G) \leq\left(1-\delta^{\prime}\right) \Delta$, for some linear function $\delta^{\prime}=\delta^{\prime}(\epsilon)>0$, where $\delta^{\prime}$ can be taken to be $\frac{\epsilon}{2}$ for $\epsilon$ sufficiently small. The techniques used in this paper, are similar to those introduced in [21].

If the neighbourhood of each vertex of $G$ had at most $(1-\epsilon)\left(\begin{array}{l}\Delta \\ 2\end{array}\right)$ edges, then we would be able to complete our total colouring of $G$ in a manner similar to that in [18]. Of course, this is not always the case. However, we can make use of a partition, introduced in [21], of $V(G)$ into $S_{1}, \ldots, S_{\ell}, H$, such that each vertex of $H$, has at most $(1-\epsilon)\left(\begin{array}{c}\Delta \\ 2\end{array}\right)$ edges in its neighbourhood, and for each $i$, the number of edges across the cut $\left(S_{i}, G-S_{i}\right)$ is small.

We will generate our vertex colourings in a random fashion. Several times in this paper, we will make use of the following tools of the Probabilistic Method.

The Local Lemma [8] Suppose $\mathcal{A}=A_{1}, \ldots, A_{n}$ is a list of random events such that for each $i, \operatorname{Pr}\left(A_{i}\right) \leq p$ and $A_{i}$ is mutually independent of all but at most $d$ other events in $\mathcal{A}$. If $\mathrm{e} p(d+1)<1$ then $\operatorname{Pr}\left(\wedge_{i=1}^{n} \bar{A}_{i}\right)>0$.

The Chernoff Bound Suppose BIN $(n, p)$ is the sum of $n$ independent Bernoulli variables each occuring with probability $p$. Then for any $0<a \leq$ $\frac{1}{6} n p:$

$$
\operatorname{Pr}(|B I N(n, p)-n p|>a)<2 \mathrm{e}^{-a^{2} / 3 n p}
$$


Remark 1.3 There are stronger versions of the Chernoff Bound (see eg. [2]), but this one is strong enough for our purposes. To deal with the case $a>\frac{1}{6} n p$ it will suffice to use $\operatorname{Pr}((|B I N(n, p)-n p|>a)<\operatorname{Pr}(|B I N(n, p)-n p|>$ $\left.\frac{1}{6} n p\right)<2 \mathrm{e}^{-\frac{1}{108} n p}$.

Azuma's Inequality [3] Let $0=X_{0}, \ldots, X_{n}$ be a martingale with

$$
\left|X_{i+1}-X_{i}\right| \leq 1
$$

for all $0 \leq i<n$. Let $\lambda>0$ be arbitrary. Then

$$
\operatorname{Pr}\left[\left|X_{n}\right|>\lambda \sqrt{n}\right]<e^{-\lambda^{2} / 2} .
$$

This yields the following very useful standard corollary.

Corollary Let $Y=Y_{1}, Y_{2}, \ldots, Y_{n}$ be a sequence of random events. Let $f(Y)=f\left(Y_{1}, Y_{2}, \ldots, Y_{n}\right)$ be a random variable defined by these $Y_{i}$. If for each $i:$

$$
\max \left|\operatorname{Exp}\left(f(Y) \mid Y_{1}, Y_{2}, \ldots Y_{i+1}\right)-\operatorname{Exp}\left(f(Y) \mid Y_{1}, Y_{2}, \ldots Y_{i}\right)\right| \leq c_{i}
$$

then the probability that $|f-\operatorname{Exp}(f)|>t$ is at most:

$$
2 \exp \left(\frac{-t^{2}}{2 \sum c_{i}^{2}}\right)
$$

For more details on this corollary and an excellent discussion of Martingale arguments see either [16] or [2].

Remark 1.4 We often apply this corollary to show concentration of variables which are functions of random permutations. Typically, these applications have the following flavour. Suppose $Z$ is a function of a random permutation $\phi: W \rightarrow U$, where $W=\left\{w_{1}, \ldots, w_{n}\right\}$. We choose our permutation by selecting for each $w_{i}$ in turn, a random member of $U$ from amongst those not previously selected. We let the event $Y_{i}$ denote this choice, and note that if for all $j>i$, the maximum effect on $Z$ of swapping the values of $\phi\left(w_{i}\right)$ and $\phi\left(w_{j}\right)$ is at most $c_{i}$, then $\max \mid \operatorname{Exp}\left(f(Y) \mid Y_{1}, Y_{2}, \ldots Y_{i+1}\right)-\operatorname{Exp}(f(Y) \mid$ $\left.Y_{1}, Y_{2}, \ldots Y_{i}\right) \mid \leq c_{i}$, and so Azuma's Inequality applies. 
Many times throughout the paper, we need to simplify expressions involving terms of the form $\left(\begin{array}{l}n \\ k\end{array}\right)$. The following inequality, which is easily verified will be very useful:

$$
\left(\begin{array}{l}
n \\
k
\end{array}\right) \leq\left(\frac{e n}{k}\right)^{k}
$$

If $A, B$ are disjoint subsets of the vertices of a graph $G$, we denote by $E_{G}(A, B)$ or $E(A, B)$ the set of edges of $G$ with one endpoint in each of $A, B$. We denote by $\bar{G}$ the complement of a graph $G$, and for any subgraph $H \subseteq G$, we denote by $\bar{S}$ the complement of $H$. If $S$ is a subset of the vertex set of $G$, then when no confusion is possible we sometimes abuse notation and use " $S$ " to also refer to the subgraph induced by $S$. Thus $\bar{S}$ refers to the complement of the subgraph induced by $S$. For any vertex $v, N(v)$ is the neighbourhood of $v$ and for each $S \subseteq V(G), \operatorname{deg}_{S}(v)=N(v) \cap S$. If for each $w$ in a ground set $W$, we have a set $F(w) \subset U$, then for each $u \in U$, we denote by $F^{-1}(u)$ the set $\{w \in W: u \in F(w)\}$.

Throughout the paper, we assume $G$ to be $\Delta$-regular, since it is straightforward to show that any graph with maximum degree $\Delta$ is a subgraph of a $\Delta$-regular graph (see for example [21]). We only claim statements to be valid for sufficiently large $\Delta$. We often use the notation $\theta(X)$ to mean an expression which is asymptotic to $z X$ where $z$ is some implicit positive constant. In general, for the sake of presentation, we omit \lfloor\rfloor and \lceil\rceil signs. All logarithms have base 2 .

Throughout this paper, we introduce several new terms. For the aid of the reader, we provide an index at the end of the paper.

\section{Isolating the Trouble-makers.}

In this section we partition the vertices of $G$ into $S_{1}, \ldots, S_{\ell}, H$, such that (a) each vertex of $H$, has relatively few edges in its neighbourhood, (b) each $S_{i}$ is very nearly a clique, and (c) for each $i$, the number of edges across the cut $\left(S_{i}, G-S_{i}\right)$ is small. This partition was introduced in [21]. We include the details here for completeness. 


\section{$2.1 \quad$ Dense Sets}

We set $\epsilon=10^{-6}$, and we call a vertex $x \in V(G)$ dense if $|E(N(x))|>$ $(1-\epsilon)\left(\begin{array}{l}\Delta \\ 2\end{array}\right)$. Otherwise $x$ is sparse.

Given a dense vertex $x$, we can recursively define a (unique) set $S_{x} \subseteq$ $V(G)$ as follows. Initially set $S_{x}=N(x) \cup\{x\}$, and then

1. delete from $S_{x}$ any vertex $y \in S_{x}$ with $\left|N(y) \cap S_{x}\right|<\frac{3 \Delta}{4}$, until no such $y$ remains, and then

2. add to $S_{x}$ any vertex $y \notin S_{x}$ with $\left|N(y) \cap S_{x}\right|>\frac{3 \Delta}{4}$, until no such $y$ remains.

It is not hard to verify (see [21]) that $S_{x}$ is uniquely defined, i.e. that it does not depend on the order in which vertices are deleted or added, and that for any $v \in S_{x}$ or $u \notin S_{x},\left|N(v) \cap S_{x}\right| \geq \frac{3 \Delta}{4}$ and $\left|N(u) \cap S_{x}\right|<\frac{3 \Delta}{4}$.

We refer to $S_{x}$ as a dense set. The following basic facts about the structure of $S_{x}$ will prove to be useful:

Lemma 2.1 (a) $\left|N(x)-S_{x}\right|<5 \epsilon \Delta$ and $\left|S_{x}-N(x)\right|<2 \epsilon \Delta$,

(b) $\Delta-5 \epsilon \Delta<\left|S_{x}\right|<\Delta+2 \epsilon \Delta$,

(c) $x \in S_{x}$,

(d) $\left|E\left(G-S_{x}, S_{x}\right)\right|<4 \epsilon \Delta^{2}$, and

(e) $\left|E\left(\overline{S_{x}}\right)\right|<\epsilon \Delta^{2}$.

Proof Suppose $N(x)-S_{x}=\left\{u_{1}, \ldots, u_{s}\right\}$ where the vertices are listed in the order that they are removed from $S_{x}$. Then $\operatorname{deg}_{N(x)}\left(u_{i}\right)<\frac{3 \Delta}{4}+(i-1)$, and so since $|E(N(x))|>\left(\begin{array}{c}\Delta \\ 2\end{array}\right)-\frac{\epsilon}{2} \Delta^{2}$ we have that $\frac{1}{2}\left(\frac{s \Delta}{4}-\frac{s(s-1)}{2}\right)<\frac{\epsilon}{2} \Delta^{2}$, and so $s<5 \epsilon \Delta$. Similarly, if $S_{x}-N(x)=\left\{v_{1}, \ldots, v_{t}\right\}$ where the vertices are listed in the order that they are added to $S_{x}$, then $\operatorname{deg}_{N(x)}\left(v_{i}\right)>\frac{3 \Delta}{4}-(i-1)$, and so $\frac{1}{2}\left(\frac{3 t \Delta}{4}-\frac{t(t-1)}{2}\right)<\frac{\epsilon}{2} \Delta^{2}$, yielding $t<2 \epsilon \Delta$ (in fact $t<1.5 \epsilon \Delta$ ) and thus proving (a).

(b) and (c) are merely trivial corollaries of (a), but worth stating, nevertheless.

Since $x$ is dense, $|E(G-N(x), N(x))|<\epsilon \Delta^{2}$. Every time a vertex is removed from $S_{x}$, it increases the number of edges across this cut by at most 
$\frac{\Delta}{2}$. Each time a vertex is added to $S_{x}$, it decreases the number of edges across this cut, and so (d) follows from (a).

(e) follows by noting that $\left|E\left(\overline{S_{i}}\right)\right| \leq|E(\overline{N(x)})|+\left(\begin{array}{l}t \\ 2\end{array}\right)+\frac{t \Delta}{4}$ and recalling that we have shown $t<1.5 \epsilon \Delta$.

As we will now see, we can partition $V(G)$ into a sequence of dense sets, and a sparse set containing no dense vertices, such that there are very few edges between sets.

Lemma 2.2 Let $G$ be any $\Delta$-regular graph. We can partition $V(G)$ into $H, S_{1}, S_{2}, \ldots, S_{\ell}$ such that

(a) for each $1 \leq i \leq \ell, S_{i}=S_{x}$ for some dense vertex $x$, and

(b) for each dense vertex $x, x \in S_{i}$ for some $i$.

Proof To prove (a) and (b), it is enough to show that if $x, y$ are both dense, then either $S_{x} \cap S_{y}=\emptyset$ or $y \in S_{x}$ (and so by symmetry $x \in S_{y}$ ). Suppose the contrary, i.e. $y \notin S_{x}$ and $S_{x} \cap S_{y} \neq \emptyset$, and consider any $a \in$ $S_{x} \cap S_{y}$.

From Lemma 2.1(a), the size of the symmetric difference of $S_{x}$ and $N(x)$ is at most $7 \epsilon \Delta$. Thus, $a$ sees at least $\frac{3 \Delta}{4}-7 \epsilon \Delta$ vertices of $N(x)$, and also of $N(y)$. Therefore $|N(x) \cap N(y)|>\frac{\Delta}{2}-14 \epsilon \Delta>\frac{\Delta}{4}$.

Also, $|N(x) \cap N(y)|<\frac{3 \Delta}{4}+7 \epsilon \Delta$, since $y \notin S_{x}$, and so $|N(x)-N(y)|>\frac{\Delta}{8}$.

Therefore, if there are at least $\frac{\Delta^{2}}{64}$ edges from $N(x) \cap N(y)$ to $N(x)-N(y)$ then $x$ is not a dense vertex, and if there are at most $\frac{\Delta^{2}}{64}$ such edges, then $y$ is not a dense vertex, yielding a contradiction.

We refer to an edge $(v, u)$, with $v \in S_{i}, u \notin S_{i}$ as an external edge of $S_{i}$ from $v$, and we refer to an edge of $E\left(S_{i}, S_{i}\right)$ as a internal edge of $S_{i}$. For each $v \in S_{i}$, we define the external neighbours and the internal neighbours of $v$ to be those vertices which are joined to $v$ by external edges and internal edges, respectively.

For each vertex $v$ in a dense set $S_{i}$, we define $O u t_{v}$ to be the set of external neighbours of $v$, i.e. the neighbours of $v$ outside of $S_{i}$. Note that $\mid$ Out $_{v} \mid \leq \frac{\Delta}{4}$ by the construction of $S_{i}$. 
Now we will partition each $S_{i}$ into $\Delta+1$ colour classes. We will not assign colours to them until later. As each $S_{i}$ is very dense, most of the colour classes will have size 1 . Unless $S_{i}$ is a clique, a few will have size 2. None will have size larger than 2.

Lemma 2.3 For each $1 \leq i \leq \ell$, we can find a matching $M_{i}$ in $\overline{S_{i}}$ such that either

(a) $M_{i}$ is a maximum matching and $\left|M_{i}\right|<10 \epsilon \Delta$, or

(b) $\left|M_{i}\right|=10 \epsilon \Delta$,

and, in either case, there is no edge $x y$ of $M_{i}$ with both $\left|N(x) \cap S_{i}\right|$ and $\left|N(y) \cap S_{i}\right|$ less than $\Delta-4 \sqrt{\epsilon} \Delta$.

Proof $\quad$ Let $A=\left\{v \in S_{i}:\left|N(v) \cap S_{i}\right|<\Delta-4 \sqrt{\epsilon} \Delta\right\}$. By Lemma 2.1, $|A|<\sqrt{\epsilon} \Delta$, and each $v \in A$ has degree at least $4 \sqrt{\epsilon} \Delta-5 \epsilon \Delta \geq 3 \sqrt{\epsilon} \Delta$ in $\overline{S_{i}}$.

Consider a maximum matching $M^{\prime}$ of $S_{i}$. If $\left|M^{\prime}\right|<10 \epsilon \Delta$ then setting $M_{i}=M^{\prime}$ satisfies our requirements, for otherwise there exists an edge $x y \in$ $M^{\prime}$ with $x, y \in A$. $x, y$ each must have at least $3 \sqrt{\epsilon} \Delta-20 \epsilon \Delta>2$ neighbours in $\overline{S_{i}}$ not covered by $M^{\prime}$ which contradicts its maximality.

If $\left|M^{\prime}\right| \geq 10 \epsilon \Delta$ and $M^{\prime}$ does not contain at least $10 \epsilon \Delta$ edges, none of which have both endpoints in $A$, then $\left|M^{\prime}\right|<10 \epsilon \Delta+\left(\begin{array}{c}|A| \\ 2\end{array}\right)<11 \epsilon \Delta$. Furthermore, $M^{\prime}$ must contain an edge $x y$ with $x, y \in A$. Again, $x, y$ each must have at least $3 \sqrt{\epsilon} \Delta-22 \epsilon \Delta>2$ neighbours in $\overline{S_{i}}$ not covered by $M^{\prime}$, contradicting its maximality. Therefore, $M^{\prime}$ must have at least $10 \epsilon \Delta$ edges, none of which have both endpoints in $A$, and so we can take $M_{i}$ to be $10 \epsilon \Delta$ of those edges.

We define the colour classes $\mathcal{C}_{i}$ of $S_{i}$ to be the set of all pairs of vertices which form an edge in $M_{i}$ along with the set of all vertices not covered by $M_{i}$. Thus, $S_{i}$ has $\left|S_{i}\right|-\left|M_{i}\right|$ colour classes. It will be convenient to occasionally also refer to each vertex of $H$ as a colour class of size 1 .

It is important to note the following:

Fact: Each colour class of a dense set has at most $\left(\frac{1}{4}+4 \sqrt{\epsilon}\right) \Delta$ external neighbours. 
Proof This follows easily from Lemma 2.3(b) and the fact that no vertex in a dense set has more than $\frac{\Delta}{4}$ external neighbours.

Lemma 2.4 For each i, $\Delta-15 \epsilon \Delta \leq\left|\mathcal{C}_{i}\right| \leq \Delta+1$.

Proof That $\left|\mathcal{C}_{i}\right| \geq \Delta-15 \epsilon \Delta$ is a simple corollary of Lemmas 2.1 and 2.3 since $\left|\mathcal{C}_{i}\right|=\left|S_{i}\right|-\left|M_{i}\right|$.

If $\left|\mathcal{C}_{i}\right|>\Delta+1$ then since $\left|\mathcal{C}_{i}\right|=\left|S_{i}\right|-\left|M_{i}\right|$, we have $\left|M_{i}\right|<\left|S_{i}\right|-(\Delta+1)<$ $10 \epsilon \Delta$, and so $M_{i}$ is a maximum matching. Consider the Tutte reduction of $\overline{S_{i}}$, i.e. a partition of $S_{i}$ into sets $X, U_{1}, U_{2}, \ldots, U_{t}$, where $|X| \leq\left|M_{i}\right|$, each $U_{j}$ is a component of $\overline{S_{i}}-X$, and at least $\left|S_{i}\right|-2\left|M_{i}\right|+|X|$ of these components are odd (see eg. [17]).

Because $\left|M_{i}\right|<\frac{1}{3}\left|S_{i}\right|$, there is at least one $U_{j}$ of size 1 . In $S_{i}$, the vertex in this component is adjacent to every other vertex in $S_{i}-X$, and so $\left|S_{i}\right|-$ $|X| \leq \Delta+1$. But $|X| \leq\left|M_{i}\right|$ and so this contradicts the assumption that $\left|\mathcal{C}_{i}\right|=\left|S_{i}\right|-\left|M_{i}\right|>\Delta+1$.

\subsection{Ornery Sets}

If $\left|\mathcal{C}_{i}\right|>\Delta-\log ^{4} \Delta$, then we say that $S_{i}$ is ornery. For each ornery set $S_{i}$, we define the kernel, $K_{i}$ of $S_{i}$ to be the set of vertices in $S_{i}$ with at most $\log ^{6} \Delta$ neighbours in $G-S_{i}$.

Lemma 2.5 For each ornery $S_{i}$,

(a) $\left|S_{i}\right|<\Delta+\log ^{5} \Delta$,

(b) $\left|S_{i}-K_{i}\right|<\log ^{5} \Delta$, and

(c) $\left|E\left(S_{i}, G-S_{i}\right)\right|<\Delta \log ^{7} \Delta$.

Proof If $S_{i}$ is ornery, then $\left|S_{i}\right|-\left|M_{i}\right|=\left|\mathcal{C}_{i}\right|>\Delta-\log ^{4} \Delta$, so by Lemma 2.1(a) we have $\left|M_{i}\right|<3 \epsilon \Delta$, and so $M_{i}$ is a maximum matching. As in the proof of Lemma 2.4, we consider the Tutte reduction of $\overline{S_{i}}$. Note that $\left|M_{i}\right|=|X|+\sum_{j=1}^{t}\left\lfloor\frac{\left|U_{j}\right|}{2}\right\rfloor$. 
Since $S_{i}$ is ornery, $\left|S_{i}\right|-\left|M_{i}\right|>\Delta-\log ^{4} \Delta$. As before, there is at least one $U_{j}$ of size 1 and so again we have $\left|S_{i}\right|-|X| \leq \Delta+1$. Also, $\left|S_{i}\right|-\left|M_{i}\right|=\left|S_{i}\right|-|X|-\sum_{j}\left\lfloor\frac{\left|U_{j}\right|}{2}\right\rfloor$. Therefore, $\sum_{j}\left\lfloor\frac{\left|U_{j}\right|}{2}\right\rfloor \leq \log ^{4} \Delta+1$ and so $\sum\left|U_{j}\right|>1\left\lceil\frac{\left|U_{j}\right|}{2}\right\rceil \leq 2 \log ^{4} \Delta+2$.

Furthermore, $\Delta-\log ^{4} \Delta \leq\left|\mathcal{C}_{i}\right| \leq \sum_{j}\left\lceil\frac{\left|U_{j}\right|}{2}\right\rceil$. Therefore the number of singleton components of $\overline{S_{i}}-X$ is at least $\Delta-3 \log ^{4} \Delta-2>\Delta-4 \log ^{4} \Delta$. Thus, by the construction of $S_{i}$, each member of $X$ must be adjacent to at least $\frac{2}{3}$ of these singleton components, and so at least one of them is adjacent to at least $\frac{2}{3}$ of $X$. Therefore, its degree is at least $\Delta-4 \log ^{4} \Delta+\frac{2}{3}|X|$, and so $|X|<6 \log ^{4} \Delta$.

(a) now follows from the fact that the vertex in any singleton component has degree at least $\left|S_{i}-X\right|-1$ in $G$, since it is adjacent to every vertex in a different component $C_{j}$.

(b) follows from the fact that each member of $S_{i}-X$ has degree at least $\Delta-\log ^{4} \Delta$ within $S_{i}$, and so $S_{i}-X \subseteq K_{i}$. (c) is a straightforward corollary of (b).

For each ornery $S_{i}$, we define Overused ${ }_{i}$ to be the set of colours which appear on at least $\Delta-\frac{\Delta}{\log ^{10} \Delta}$ edges of $E\left(S_{i}, G-S_{i}\right)$. When we eventually colour the vertices of $S_{i}$, we will not use any colours from Overused $_{i}$. In order to be able to do this, we need the following lemma.

Lemma 2.6 For any ornery $S_{i},\left|\mathcal{C}_{i}\right| \leq \Delta-$ Overused $_{i}+1$.

Proof Again, we consider the Tutte reduction of $\overline{S_{i}}$. Recall from the previous proof that there are at least $\Delta-4 \log ^{4} \Delta$ components $C_{j}$ of size 1 .

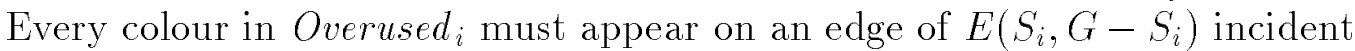
to at least $\Delta-\frac{\Delta}{\log ^{10} \Delta}-\left(\left|S_{i}\right|-\left(\Delta-4 \log ^{4} \Delta\right)\right)$ of these components, and by Lemma 2.5(a) this number is at least $\Delta-\frac{\Delta}{2 \log ^{10} \Delta}$. Furthermore, by Lemma $2.5(\mathrm{c}), \mid$ Overused $_{i} \mid<2 \log ^{7} \Delta$, and so the number of these components for which at least one colour in Overused $i$ does not appear on an external edge from that component is at most $\frac{\Delta}{2 \log ^{10} \Delta} \times 2 \log ^{7} \Delta<\Delta-4 \log ^{4} \Delta$. Therefore, there is at least one singleton component which is incident to an external edge

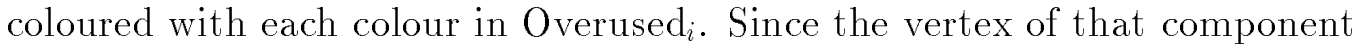
is also adjacent to every other vertex in $S_{i}-X$, we have $\Delta \geq\left|S_{i}\right|-|X|+$ $1+$ Overused $_{i}$, and the lemma follows from the fact that $|X| \leq\left|M_{i}\right|$ and 
$\left|\mathcal{C}_{i}\right|=\left|S_{i}\right|-\left|M_{i}\right|$.

Corollary 2.7 For any ornery set $S_{i}, \mid$ Overused $_{i} \mid<\log ^{4} \Delta+1$.

Proof This follows from Lemma 2.6 and the fact that if $S_{i}$ is ornery then $\left|\mathcal{C}_{i}\right|>\Delta-\log ^{4} \Delta$.

\subsection{Modifications to $G$}

When colouring an ornery set $S_{i}$, colours which appear on Out for too many values of $v$ will cause difficulties. In order to prevent such difficulties, we will make some modifications to $G$.

For each ornery set $S_{i}$, we define $B i g_{i}=\left\{v \notin S_{i}:\left|N(v) \cap S_{i}\right|>\Delta^{7 / 8}\right\}$.

Modification 1: We add edges to make each $\mathrm{Big}_{i}$ a near clique. That is, for any pair of non-adjacent vertices $x, y \in \mathrm{Big}_{i}$, not both in the same dense set, we add the edge $(x, y)$. This will ensure that no colour can appear on two vertices in $\mathrm{Big}_{i}$, and so if a colour appears on $\mathrm{Out}_{v}$ for several (say greater than $\frac{9 \Delta}{10}$ ) vertices $v \in S_{i}$, then that colour must appear on many vertices in $G-S_{i}-\mathrm{Big}_{i}$.

A similar problem arises if a colour appears on too many edges out of $S_{i}$ in the initial edge-colouring.

For each ornery $S_{i}$, we define Moderate $_{i}$ to be the set of colours which appear on at least $\Delta / \log \Delta$ and at most $\Delta-\Delta / \log ^{10} \Delta$ external edges of $S_{i}$.

Modification 2: We prevent each $\mathrm{Big}_{i}$ from receiving any colours in Moderate $_{i}$. That is, for each colour $c \in$ Moderate $_{i}$, we add a new vertex to $G$, and add edges from it to every vertex in $\mathrm{Big}_{i}$. We colour this vertex with colour $c$.

Through a slight abuse of notation, we continue to refer to the modified graph as $G$. We refer to the new edges and vertices as artificial. These modifications will be most important in Section 6.1. At the beginning of Section 6.2 all artificial vertices and edges are removed, and $G$ is returned to its original state.

These modifications may alarm the reader, as they cause the maximum degree of $G$ to increase. However, this will not pose a problem. For one thing, 
the artificial edges will not be coloured in our total colouring. Furthermore, the degrees of the vertices increase just slightly, so little in fact, that we will still be able to find a suitable $\Delta+1$ colouring of $G$. Also every vertex which was originally sparse will still be nearly sparse.

Lemma 2.8 (a) for each $v \in G, \operatorname{deg}(v) \leq \Delta+\sqrt{\Delta}$;

(b) for each $v$ in a dense set $S_{i},\left|\mathrm{Out}_{v}\right| \leq \frac{\Delta}{4}+\sqrt{\Delta}$;

(c) for each $v \notin S_{i},\left|N(v) \cap S_{i}\right| \leq \frac{3 \Delta}{4}+\sqrt{\Delta}$;

(d) for each colour class $X \in S_{i}$, the number of external edges from $X$ is at most $\frac{\Delta}{3}$;

(e) for each dense set $S_{i}$, the number of external edges from $S_{i}$ is at most $5 \epsilon \Delta^{2}$;

(f) for each ornery $S_{i}$, the number of external edges from $S_{i}$ is at most $\Delta \log ^{7} \Delta+\sqrt{\Delta}$

(g) for each ornery $S_{i}$, and each $v \in K_{i},\left|\mathrm{Out}_{v}\right| \leq \log ^{6} \Delta$;

(h) for each ornery $S_{i}$, and each $v \notin \operatorname{Big}_{i},\left|N(v) \cap S_{i}\right| \leq \Delta^{7 / 8}+\sqrt{\Delta}$;

(i) for each sparse vertex $v,|E(N(v))| \leq\left(1-\frac{\epsilon}{2}\right)\left(\begin{array}{c}\Delta \\ 2\end{array}\right)$.

\section{Proof}

(a) By Lemma 2.5(c), for each $i, \mid$ Big $_{i} \mid \leq \Delta^{1 / 8} \log ^{7} \Delta$. Furthermore, $v$ lies in at most $\Delta^{1 / 8}$ different Big $_{i}$ 's. Thus the number of artifical edges incident to $v$ is at most $\Delta^{1 / 4} \log ^{7} \Delta \leq \sqrt{\Delta}$.

(b)-(f), (h),(i) follow in a similar manner. (g) follows from the fact that if $v \in K_{i}$ then $\mid$ Out $_{v} \mid \leq \log ^{6} \Delta$ before the modifications, so $v \notin$ Big $_{j}$ for any $j$, and so there are no artificial edges incident to $v$.

\subsection{The Neighbourhoods of the Sparse Vertices}

Recall that since $H=V(G)-\cup_{i=1}^{\ell} S_{i}$, every vertex in $H$ is sparse. There may also be sparse vertices in the dense sets. Define $H^{\prime}=\{v \in V(G)-H$ : $\left.\left|\mathrm{Out}_{v}\right|>\frac{1}{10^{4}} \Delta\right\}$. Note that every vertex in $H^{\prime}$ is sparse, as by Lemma 2.8(e) there are at least $\left|\mathrm{Out}_{v}\right| \times \frac{3 \Delta}{4}-5 \epsilon \Delta^{2} \geq \epsilon \Delta^{2}$ missing edges between the internal neighbours and the external neighbours of $v$. 
We say that two vertices are strongly non-adjacent if there are no edges between their colour classes, and if they do not both lie in the same dense set. Thus two vertices are eligible to receive the same colour if they are strongly non-adjacent. When we colour $G$, we will try to use several colours twice in the neighbourhood of any $v \in H \cup H^{\prime}$, and so we need to show that each such vertex has several pairs of strongly non-adjacent neighbours.

Lemma 2.9 For any $v \in H \cup H^{\prime}, N(v)$ has at least $\frac{\epsilon}{80} \Delta^{2}$ pairs of strongly non-adjacent neighbours.

\section{Proof}

Case 1: $v \in H^{\prime}$.

$v$ has at least $\frac{3 \Delta}{4}$ internal neighbours and $\frac{1}{10^{4}} \Delta$ external neighbours. Every external edge from $S_{i}$ causes at most 4 pairs consisting of one internal and one external neighbour to not be strongly non-adjacent. Thus, by Lemma $2.8(\mathrm{e})$, the number of such strongly non-adjacent pairs is at least $\frac{3 \Delta}{4}\left(10^{-4} \Delta\right)-$ $20 \epsilon \Delta^{2}>\epsilon \Delta^{2}$.

Case 2: $v \in H$.

Let $w_{i}=\left|N(v) \cap S_{i}\right|$, and $W=\sum_{i=1}^{\ell} w_{i}$. By Lemma 2.8(i), there are at least $\frac{\epsilon}{4} \Delta^{2}$ pairs of non-adjacent vertices in $N(v)$. If at least $\frac{\epsilon}{8} \Delta^{2}$ of them lie entirely within $H$, then we are done. Otherwise, by Lemma 2.8(a), we must have $(\Delta+\sqrt{\Delta}) W>\frac{\epsilon}{8} \Delta^{2}$, and so $W>\frac{\epsilon}{10} \Delta$.

If for any $i, w_{i} \geq \sqrt{\epsilon}$, then as in Case 1 , we have at least $\left(\frac{\sqrt{\epsilon}}{4}-20 \epsilon\right) \Delta^{2}>$ $\epsilon \Delta^{2}$ strongly non-adjacent pairs.

If $w_{i}<\sqrt{\epsilon} \Delta$ for all $i$, then by Lemma $2.8(\mathrm{~d})$, each $v \in S_{i}$ is strongly non-adjacent to at least $\Delta-w_{i}-2\left(\frac{\Delta}{3}\right)>\frac{\Delta}{4}$ vertices of $N(v)-S_{i}$, and so $N(v)$ has at least $\frac{1}{2} W \times \frac{\Delta}{4}>\frac{\epsilon}{80} \Delta^{2}$ pairs of strongly nonadjacent vertices.

\section{Colouring the Vertices - An Overview}

Recall that we begin with an arbitrary proper $\Delta+1$ edge colouring of $G$, and our goal is to show that there exists a proper $\Delta+1$ vertex colouring of $G$ which does not conflict with the edge colouring too much.

Given the union of a proper edge colouring and a proper vertex colouring of $G$ (where the two proper colourings possibly conflict with each other), we 
define the reject edges of $G$ to be the set of edges which have the same colour as one of their endpoints. For any vertex $v \in V(G)$, we define the reject degree of $v$ to be the number of reject edges incident to $v$, not counting the (at most one) such edge with the same colour as $v$. We define the reject graph to be the subgraph of $G$ induced by the reject edges. Note that for any vertex $v$, the degree of $v$ in the reject graph is at most the reject degree of $v$ plus 1 .

We will show that given any proper $\Delta+1$ edge colouring, we can find a proper $\Delta+1$ vertex colouring such that the maximum reject degree is $C-3$. Thus, we will be able to recolour the edges in $R$ using $C-1$ new colours, providing a $\Delta+C$ total colouring of $G$.

We will find our vertex colouring using a random colouring procedure. We will prove, using various tools of the probabilistic method, that with positive probability our procedure succeeds in finding a satisfactory colouring. Our procedure consists of four main phases:

Phase 1: An Initial Colouring.

We assign an initial random colour to nearly every vertex. To do this, we assign to each vertex in $H$ a uniformly random colour, and we assign a random permutation of colours to the colour classes of each dense set.

There will almost certainly be some conflicts. We resolve some of them by uncolouring a vertex in $H$ if it has a neighbour of the same colour. All remaining conflicts are between vertices in different dense sets. We consider these vertices to be only temporarily coloured, and we will recolour them during Phase 3.

We also uncolour vertices if they cause the reject degree of a neighbour to grow too high.

Phase 2: Finishing the Dense Sets.

We randomly colour all the vertices in dense sets which did not retain a colour in the first round. We ensure that the reject degree within the dense sets does not grow too high.

If a vertex receives the same colour as one of its external neighbours or one of its external edges, then we consider it to be temporarily coloured, and we will recolour it during Phase 3 . Note that this prevents the appearance of any external reject edges.

Phase 3: Recolouring the Temporarily Coloured Vertices.

We recolour all the temporarily coloured vertices other than those in $H^{\prime}$. We divide them into three groups: 


\section{A) The kernels of the ornery sets.}

These require the most work. We will show that there are very few such vertices to be recoloured, and that we will be able to swap the colour of each such vertex with another vertex in the dense set. These other vertices will always be available because of the very small number of external edges from an ornery set.

B) Vertices in non-ornery dense sets with external degree at most $\log ^{3} \Delta$.

We will ensure that there are at most $50 \log ^{3} \Delta$ such temporarily coloured vertices in any dense set. Since any non-ornery dense set has at most $\Delta-$ $\log ^{4} \Delta$ colour classes, we will be able to recolour these vertices using the $\log ^{4} \Delta$ colours not yet used on that dense set.

C) Vertices with external degree between $\log ^{3} \Delta$ and $\frac{1}{10^{4}} \Delta$

We will see that each such vertex has enough colours appearing twice in its neighbourhood that we will be able to colour them in a greedy manner, always preventing the reject degrees from growing too high.

Phase 4: Finishing the Sparse Vertices.

At this point, all that remains is to colour the vertices of $H$ which did not retain their colours in Phase 1, and the temporarily coloured vertices of $H^{\prime}$. We will show that each of these vertices has at least $\theta(\Delta)$ repeated colours in its neighbourhood, and this will allow us to colour them all while keeping the reject degrees low.

During Phase $i$, we keep the reject degree of any vertex from increasing by more than $C_{i}, i=1, \ldots, 4$. Again, we make no attempt to optimize each $C_{i}$. It suffices to take $C_{1}=4000, C_{2}=1700, C_{3}=2000$, and $C_{4}=10^{25}$, and so when the vertex colouring is completed, the reject degree of each vertex will be at most $C_{1}+C_{2}+C_{3}+C_{4}<C-3$ where $C=10^{26}$.

In the following sections, we will elaborate on each of these phases, and prove that they can each be successfully completed.

\section{Phase 1: An Initial Colouring.}

In this phase we assign a random colour to each vertex of $G$, and then uncolour some of the vertices when either conflicts occur or the reject degree is too high. In order to facilitate Phase 2, we choose a small random subset of the vertices in each dense set which we will not colour during this phase. This will give us better control over the distribution of the uncoloured vertices at 
the end of the phase.

We obtain our initial colouring through the following procedure:

1. Assign a random colour to most vertices in $G$ using the following procedure.

\section{COL:}

(a) Assign to each $v \in H$ a colour chosen uniformly at random from $\{1, \ldots, \Delta+1\}$.

(b) For each dense set $S_{i}$, choose a set $A_{i}$ of $\left|\mathcal{C}_{i}\right|$ colours uniformly at random from $\{1, \ldots, \Delta+1\}-$ Overused $_{i}$ (where we consider Overused $_{i}=\emptyset$ if $S_{i}$ is not ornery). Then take uniformly random subsets $\mathcal{C}_{i}^{\prime} \subset \mathcal{C}_{i}$ and $A_{i}^{\prime} \subset A_{i}$, each of size $\left|\mathcal{C}_{i}\right|-\gamma \Delta$, where $\gamma=\frac{1}{5000}$, and assign a uniformly random permutation of $A_{i}^{\prime}$ to the colour classes $\mathcal{C}_{i}^{\prime}$.

2. If a vertex $v \in H$ receives the same colour as one of its neighbours, then we uncolour $v$.

3. If a vertex $v \in G$ has reject degree at least $C_{1}$, then we uncolour every vertex $w \in N(v)$ such that $w$ received the same colour as the edge $(w, v)$.

At this point we will have produced a partial colouring such that the reject degree is at most $C_{1}$. This will not neccessarily be a proper colouring as there may be some vertices in the dense sets, which have the same colour as a neighbour in another dense set. We correct this as follows:

If a vertex $v \in S_{i}$ retains the same colour as a neighbour in $S_{j}, j \neq i$, then we consider $v$ to be temporarily coloured. All other vertices in $G$ which retain their colours are considered to be truly coloured. The colour on a truly coloured vertex is said to appear truly on that vertex. We define Temp $i$ to be the set of temporarily coloured vertices of $S_{i}$. For each $1 \leq a \leq \frac{1}{4} \Delta+\sqrt{\Delta}$, we define $\operatorname{Temp}_{i}(a)$ to be the subset of $\operatorname{Temp}_{i}$ with external degrees at most $a$.

For each dense set $S_{i}$, we define $W_{i}$ to be the set of uncoloured (i.e. neither truly coloured nor temporarily coloured) colour classes of $S_{i}$, and $U_{i}$ to be the unused colours from $A_{i}$. Note that $\left|W_{i}\right|=\left|U_{i}\right|$. In Phase 2, we will match 
the colours of $U_{i}$ to $W_{i}$. For each $v \in S_{i}$, we define the potential reject edges incident to $v, \operatorname{PR}(v)$ to be the set of edges between $v$ and $W_{i}$ whose colour is in $U_{i}$.

In order for the later stages to be successful, we need the following properties to hold for our partial colouring:

(P1.1) For each $v \in H \cup H^{\prime}, N(v)$ has at least $\frac{\epsilon \gamma^{3}}{4} \Delta$ colours which appear truly twice.

(P1.2) For each $v \in G-\left(H \cup H^{\prime}\right)$ such that $\left|\mathrm{Out}_{v}\right| \geq \log ^{3} \Delta$, the number of colours that appear truly in both Out and $_{v}(v) \cap S_{i}$ exceeds the number of colours on external edges of $v$ which do not appear truly on $N(v)$ by at least $\frac{1}{200}\left|\mathrm{Out}_{v}\right|$.

(P1.3) For each $i$ and $a \geq \log ^{3} \Delta,\left|\operatorname{Temp}_{i}(a)\right| \leq 2 a$.

(P1.4) For each ornery $S_{i}$, and each colour $c$, the number of vertices in $S_{i}$ having an external neighbour outside of $\mathrm{Big}_{i}$ with colour $c$ is at most $\Delta^{31 / 32}$.

(P1.5) For each $i,\left|W_{i}\right| \leq \gamma \Delta+\frac{1}{10^{6}} \Delta$.

(P1.6) For each $i$, and each $v \in S_{i},|P R(v)| \leq 3 \gamma^{2} \Delta$.

We prove that these properties all hold with positive probability via the following sequence of lemmas.

Lemma 4.1 Given any list of colour classes $C=C_{1}, \ldots, C_{t}$, and a corresponding list of colours $c_{1}, \ldots, c_{t}$, the probability that $C_{i}$ receives $c_{i}$ for each $i=1, \ldots, t$ is at most $\left(\frac{3}{\Delta}\right)^{t}$.

Proof For each dense set $S_{j}$, let $t_{j}$ denote the size of $C \cap \mathcal{C}_{j}$, and let $t_{0}=t-\sum_{j \geq 1} t_{j}$ denote the number of classes in $C$ which consist of a single vertex in $H$.

The probability that each $C_{i}$ receives $c_{i}$ is at most

$$
\left(\frac{1}{\Delta+1}\right)^{t_{0}} \times \prod_{j \geq 1} \frac{\left(\left|\mathcal{C}_{j}\right|-t_{j}\right) !}{\left(\left|\mathcal{C}_{j}\right|\right) !}
$$

For any $t$, this product is maximized when each $t_{j}=\left|C_{j}\right|$ or $t_{j}=0$ for all but at most one value of $j=j^{*}$. In this case, the product is at most

$$
\left(\frac{1}{\Delta+1}\right)^{t_{0}} \times \frac{\left(\left|\mathcal{C}_{j^{*}}\right|-t_{j^{*}}\right) !}{\left(\left|\mathcal{C}_{j^{*}}\right|\right) !} \times \prod_{j \neq j^{*}} \frac{1}{t_{j} !}<\left(\frac{\mathrm{e}}{(1-10 \epsilon) \Delta}\right)^{t}<\left(\frac{3}{\Delta}\right)^{t},
$$

since $\left|\mathcal{C}_{i}\right| \geq \Delta-10 \epsilon \Delta$ by Lemma 2.3 . 
Next, we will show that with high probability, only a small proportion of the colour classes get uncoloured because of high reject degrees. Denote by $R$ the set of classes uncoloured during Step 3 of Phase 1, and denote by $R^{\prime}$ the set of vertices with reject degree greater than $C_{1}$ after Step 1 of Phase 1. For each dense set $S_{i}$, denote by $Q_{i}$ the set of colours which are removed from $R \cap S_{i}$ during Step 3.

Lemma 4.2 For any set $X$ of at most $2 \Delta$ colour classes, the probability that $|X \cap R|>\frac{1}{10^{20}}|X|$ is at most $\mathrm{e}^{-\theta(|X|)}$.

Proof Consider any $X_{0} \subseteq X,\left|X_{0}\right|=\frac{1}{10^{20}}|X|$. We will bound the probability that $X_{0} \subseteq R$.

If $X_{0} \subseteq R$, then for each colour class $x \in X_{0}$, we can choose a particular $r_{x} \in R^{\prime}$ such that $x$ receives the colour appearing on an edge $e_{x}$ from $x$ to $r_{x}$. If this is the case, then we let $R_{0}^{\prime}=\left\{r_{x} \mid x \in X_{0}\right\}$.

We will first determine the probability that $X_{0} \subseteq R$ and $\left|R_{0}^{\prime}\right|<\frac{1}{100}\left|X_{0}\right|$.

There are at most $(2 \Delta)^{\left|X_{0}\right|}$ choices for the vector $\left(e_{x}\right)_{x \in X_{0}}$. Clearly, the number of such vectors which yield $\left|R_{0}^{\prime}\right|<\frac{1}{100}\left|X_{0}\right|$ is maximal when every $v \in \cup_{x \in X_{0}} x$ has the same neighbourhood, and so this number is at most $\left(\frac{1}{100}\left|X_{0}\right|\right)\left(\frac{2}{100}\left|X_{0}\right|\right)^{\left|X_{0}\right|}$. Thus, by Lemma 4.1, the probability that $X_{0} \subseteq R$ and $\left|R_{0}^{\prime}\right|<\frac{1}{100}\left|X_{0}\right|$ is at most:

$$
\left(\begin{array}{c}
\Delta \\
\frac{1}{100}\left|X_{0}\right|
\end{array}\right)\left(\frac{2}{100}\left|X_{0}\right|\right)^{\left|X_{0}\right|}\left(\frac{3}{\Delta}\right)^{\left|X_{0}\right|}<\left(\frac{1}{5 \times 10^{20}}\right)^{\frac{1}{10^{20}}\left|X_{0}\right|}
$$

since $\left|X_{0}\right|<\Delta$.

Next, we determine the probability that $X_{0} \subseteq R$ and $\left|R_{0}^{\prime}\right| \geq \frac{1}{100}\left|X_{0}\right|$.

For each $r \in R_{0}^{\prime}$, we set $d_{r}$ to be $C_{1}$ minus the number of reject edges from $r$ to $X_{0}$. Note that each $x \in X_{0}$ can contribute at most 2 such edges, and so $\sum_{r \in R_{0}^{\prime}} d_{r} \geq C_{1}\left|R_{0}^{\prime}\right|-2\left|X_{0}\right| \geq\left(\frac{C_{1}}{100}-2\right)\left|X_{0}\right|$. Each $r \in R_{0}^{\prime}$ must have at least $d_{r}$ neighbours in $G-X_{0}$ which contribute a reject edge incident with $d_{r}$. Thus, the total number of such edges from $G-X_{0}$ to $R$ must be at least $\left(\frac{C_{1}}{100}-2\right)\left|X_{0}\right|$. Each colour class can contribute at most 2 such edges and so there must be at least $\left(\frac{C_{1}}{200}-1\right)\left|X_{0}\right|$ colour classes contributing these edges.

The number of choices for these colour classes, along with the edges they contribute, is at most $\left(\begin{array}{c}\Delta\left|R_{0}^{\prime}\right| \\ \left(\frac{C_{1}}{200}-1\right)\left|X_{0}\right|\end{array}\right)$. Thus, by Lemma 4.1 the probability that there are this many reject edges from $G-X_{0}$ to $R_{0}^{\prime}$ is at most: 


$$
\left(\begin{array}{c}
\Delta\left|R_{0}^{\prime}\right| \\
\left(\frac{C_{1}}{200}-1\right)\left|X_{0}\right|
\end{array}\right)\left(\frac{3}{\Delta}\right)^{\left(\frac{C_{1}}{200}-1\right)\left|X_{0}\right|}<\left(\frac{3 \mathrm{e}}{C_{1} / 200-1}\right)^{\left(\frac{C_{1}}{200}-1\right)\left|X_{0}\right|}
$$

since $\left|R_{0}^{\prime}\right| \leq\left|X_{0}\right|$. Also, as before there are at most $(2 \Delta)^{\left|X_{0}\right|}$ choices for the vector $\left(e_{x}\right)_{x \in X_{0}}$ of reject edges from $X_{0}$ to $R_{0}^{\prime}$. Therefore the probability that $X_{0} \subseteq R$ and $\left|R_{0}^{\prime}\right| \geq \frac{1}{100}\left|X_{0}\right|$ is at most:

$$
(2 \Delta)^{\left|X_{0}\right|}\left(\frac{3}{\Delta}\right)^{\left|X_{0}\right|}\left(\frac{3 \mathrm{e}}{C_{1} / 200-1}\right)^{\left(\frac{C_{1}}{200}-1\right)\left|X_{0}\right|}<\left(\frac{1}{5 \times 10^{20}}\right)^{\frac{1}{10^{20}}\left|X_{0}\right|} .
$$

Therefore, for any choice of $X_{0}$, the probability that $X_{0} \subseteq R$ is at most $2\left(\frac{1}{5 \times 10^{20}}\right)^{\frac{1}{10^{20}}\left|X_{0}\right|}$, and so, setting $\rho=1 /\left(5 \times 10^{20}\right)$, the probability that $\mid X \cap$ $R\left|>\frac{1}{10^{20}}\right| X \mid$ is at most:

$$
2\left(\begin{array}{c}
|X| \\
\frac{1}{10^{20}}|X|
\end{array}\right)\left(\frac{1}{5 \times 10^{20}}\right)^{\frac{1}{10^{20}}|X|} \leq 2\left(\frac{\mathrm{e}|X|}{5 \rho|X|}\right)^{5 \rho|X|} \times \rho^{5 \rho|X|}=2\left(\frac{\mathrm{e}}{5}\right)^{5 \rho|X|}
$$

Lemma 4.3 For any dense set $S_{i}$, and any set of colours $U \subseteq\{1, \ldots, \Delta\}$, the probability that $\left|U \cap Q_{i}\right|>\frac{1}{10^{20}}|U|$ is at most $\mathrm{e}^{-\theta(|X|)}$.

Proof For each $X_{0} \subseteq \mathcal{C}_{i},\left|X_{0}\right|=\frac{1}{10^{20}}|U|$, we bound the probability that $X_{0} \subset R$, and $X_{0}$ receives colours from $U$. The proof follows along the same lines as the proof of Lemma 4.2, and we omit the details.

Now we will prove that with positive probability our colouring satisfies the desired properties.

Lemma 4.4 With positive probability, the partial colouring produced by Phase 1 satisfies properties (P1.1) - (P1.6). 
Proof We will use the Local Lemma. We define the following events: $A 1.1(v)$ - Defined for each $v \in H \cup H^{\prime}$, this is the event that fewer than $\frac{\epsilon \gamma^{3}}{4} \Delta$ colours appear truly twice in $N(v)$.

$A 1.2(v)$ - Defined for each vertex $v$ in $G-\left(H \cup H^{\prime}\right)$ with $\left|\mathrm{Out}_{v}\right| \geq \log ^{3} \Delta$, this is the event that the number of colours which appear truly in both Out $v$ and $N(v) \cap S_{i}$ does not exceed the number of colours on external edges of $v$ which do not appear truly in $N(v)$ by at least $\frac{1}{200}\left|\mathrm{Out}_{v}\right|$.

$A 1.3(i, a)$ - Defined for each $1 \leq i \leq \ell$ and $\log ^{3} \Delta \leq a \leq \Delta$, this is the event that $\left|\operatorname{Temp}_{i}(a)\right|>2 a$.

A1.4(i,c) - Defined for each $i$ such that $S_{i}$ is ornery and each $c \in$ $\{1, \ldots, \Delta+1\}$, this is the event that the number of vertices in $S_{i}$ having an external neighbour outside of $\mathrm{Big}_{i}$ with colour $c$ is more than $\Delta^{31 / 32}$.

A1.5(i) - Defined for each $1 \leq i \leq \ell$, this is the event that $\left|W_{i}\right|>$ $\gamma \Delta+\frac{1}{10^{6}} \Delta$.

$A 1.6(v)$ - Defined for each $v$ in any dense set $S_{i}$, this is the event that $|P R(v)|>3 \gamma^{2} \Delta$.

We wish to show that with positive probability none of these events hold.

For each vertex $v \in G$, define $N^{3}(v)$ to be the set of vertices of distance at most 3 from $v$, and set $D(v)=N^{3}(v) \cup\left\{S_{i} \mid S_{i} \cap N^{3}(v) \neq \emptyset\right\}$. For each dense set $S_{i}$, set $D\left(S_{i}\right)=\cup_{v \in S_{i}} D(v)$. For each appropriate vertex $v, A 1.1(v)$ is independent of the colour assigned to all vertices in $G-D(v)$, and the analogous bound holds for each of the other events. Thus, for example, $A 1.1(v)$ is independent of $A 1.3(i, a)$ as long as $D(v) \cap D\left(S_{i}\right)=\emptyset$. It follows that each event is independent of all but at most $\Delta^{9}$ other events. Thus, to apply the Local Lemma, it will suffice to show that each event holds with probability at most $\Delta^{-10}$.

$\operatorname{Pr}(A 1.1(v)) \leq \Delta^{-10}:$

To bound the probability of $A 1.1(v)$, we define $Z_{1}$ to be the number of colours which are retained by exactly two vertices in $N(v)$, after Step 2. If $Z_{1} \geq \frac{\epsilon \gamma^{3}}{2} \Delta$, and fewer than $\frac{\epsilon \gamma^{3}}{4} \Delta>\frac{1}{10^{20}} \Delta$ vertices in $N(v)$ are uncoloured during Step 3, then $A 1.1(v)$ does not hold. Thus, by Lemma 4.2, it is enough to bound the probability that $Z_{1}<\frac{\epsilon \gamma^{3}}{2} \Delta$.

Since $v \in H \cup H^{\prime}$, by Lemma $2.9, N(v)$ has at least $\frac{\epsilon}{80} \Delta^{2}$ pairs of strongly non-adjacent vertices $x, y$. For any particular such pair, denote by $X, Y$, the 
colour classes containing $x, y$ respectively.

By Lemma 2.4, for each $S_{i},\left|\mathcal{C}_{i}^{\prime}\right| \geq(1-15 \epsilon-\gamma) \Delta>(1-2 \gamma) \Delta$, and so $x, y$ both receive a colour in Step 1 with probability at least $(1-2 \gamma)^{2}$. Also, given that they both receive a colour, they each receive the same colour with probability at least $\frac{1}{\Delta+1}$, since they do not both lie in one dense set. It is straightforward to verify that the probability that no other vertex in $N(v) \cup N(X) \cup N(Y)$ receives that colour is at least $\gamma^{2}$ (the worst case occurs when the portion of $N(v) \cup N(X) \cup N(Y)$ which is outside of the dense sets that $X$ and $Y$ belong to lies entirely within 2 other dense sets). Thus $\operatorname{Exp}\left(Z_{1}\right) \geq\left(\frac{\epsilon}{80}\right) \Delta^{2}\left(\frac{\gamma^{2}(1-2 \gamma)^{2}}{\Delta+1}\right) \geq \epsilon \gamma^{3} \Delta$.

We now use Azuma's inequality to show that $Z_{1}$ is concentrated around its mean. We will use the sort of argument described in Remark 1.4. For the sake of this proof, we consider choosing the colouring in Step 1 in an unusual manner. We first assign an initial colouring by applying COL. Next we recolour the vertices of $N(v)$. In particular, for each $x \in N(v)$ in turn: if $x \in H$ then we recolour $x$ with a uniformly random colour from $\{1, \ldots, \Delta+1\}$; if $x$ is in some dense set $S_{i}$, then we choose a uniformly random colour $c^{\prime} \in\{1, \ldots, \Delta+1\}-$ Overused $_{i}$, and swap $c^{\prime}$ in $S_{i}$ with the colour that $x$ was originally assigned. Note that the resulting random colouring is selected from the same distribution as a colouring selected by a single application of COL, and so it is valid to generate the colouring in this manner.

For each colour class $w \in G, w \cap N(v)=\emptyset$, define $X_{w} \subseteq N(v)$ to be the set of vertices in $N(v)$ whose colour class is adjacent to $w$. Order the colour classes of each dense set $S_{i}$ starting with those not intersecting $N(v)$ in nonincreasing order of $\left|X_{w}\right|$, and finishing with those classes which intersect $N(v)$ ordered arbitrarily. We choose our random permutation in COL by assigning each class of $\mathcal{C}_{i}^{\prime}$ in order a uniformly random colour from amongst the remaining colours of $A_{i}^{\prime}$.

In order to apply Azuma's Inequality, we must bound the effect that each random choice can have on the conditional expected value of $Z_{1}$. We split these random choices into 6 categories:

(1) The colour assigned to a vertex $w \in H$, with $w \notin N(v)$ :

The choice of the colour assigned to $w$ affects the conditional expected value of $Z_{1}$ (conditioned on the choices made thus far) by at most $\Delta\left|X_{w}\right| \times \frac{1}{\Delta^{2}}$, as this is the maximum number of strongly nonadjacent pairs in $N(v)$ that $w$ is adjacent to, multiplied by the probability that both members of this pair 
receive that colour during recolouring.

(2) The colour assigned to a colour class $w \notin H, w \cap N(v)=\emptyset$ :

Here, $w \in \mathcal{C}_{i}$ for some dense set $S_{i}$, and for any other colour class $w^{\prime} \in \mathcal{C}_{i}$ which has not yet been assigned a colour, we have $\left|X_{w^{\prime}}\right| \leq\left|X_{w}\right|$. Therefore, exchanging the colours on $w$ and $w^{\prime}$ will affect at most $2\left|X_{w}\right|$ strongly adjacent pairs within $N(v)$ and so the choice of the colour assigned to $w$ affects the conditional expected value of $Z_{1}$ by at most $2 \Delta\left|X_{w}\right| \times \frac{1}{\Delta^{2}}$.

(3) The colour initially assigned to a vertex $w \in H$ with $w \notin N(v)$.

This choice for $w$ affects the conditional expectation of $Z_{1}$ by at most 8 . To see this, consider the effect of changing the initial colour on $w$. After all the recolouring is completed, there will be at most two colour classes whose colours are affected by this exchange. Each colour can contribute at most 4 to $Z_{1}$ (in the case that two colour classes of size 2 receive that colour), or subtract at most 4 from $Z_{1}$.

(4) The colour initially assigned to a colour class $w \notin H$ with $w \cap N(v) \neq \emptyset$.

This choice for $w$ affects the conditional expectation of $Z_{1}$ by at most 16. To see this, consider the effect of exchanging the initial colour on $w$ with that of some other colour class $w^{\prime}$ in the same dense set, and reason as in the previous case.

(5) The colour with which a vertex $w \in H$ with $w \cap N(v) \neq \emptyset$ is recoloured.

It is easy to see that this choice can affect $Z_{1}$ by at most 2 .

(6) The colour with which a colour class $w \notin H$ with $w \cap N(v) \neq \emptyset$ is recoloured.

This choice $w$ can affect $Z_{1}$ by at most 8 , since the new colour which $w$ receives can add or subtract at most 4 to/from $Z_{1}$, and similarly for the other vertex involved in the swap.

Now we must sum the squares of these values. Each $x \in N(v)$ lies in $X_{w}$ for at most $2 \Delta$ values of $w$. Thus, $\sum_{w}\left|X_{w}\right| \leq 2 \Delta^{2}$, and since $\left|X_{w}\right| \leq 2 \Delta$ for each $w, \sum_{w}\left(2 \Delta\left|X_{w}\right| \times \frac{1}{\Delta^{2}}\right)^{2} \leq 16 \Delta$. Thus, denoting as usual the maximum effect of the $i$ th random choice on the conditional expected value of $Z_{1}$ by $c_{i}$, we have $\sum c_{i}^{2} \leq 16 \Delta+16 \Delta+4 \Delta=36 \Delta$ where the first term comes from the choices in categories $(1,2)$, the second term from those in $(3,4)$ and the final term from those in $(5,6)$. Thus, it follows from Azuma's Inequality (see Remark 1.4) that $\operatorname{Pr}\left(Z_{1} \leq \frac{\epsilon \gamma^{3}}{2} \Delta\right) \leq \mathrm{e}^{-\theta(\Delta)}$. Therefore, by Lemma 4.2. $\operatorname{Pr}(A 1.1(v)) \leq \mathrm{e}^{-\theta(\Delta)}<\Delta^{-10}$. 


\section{$\operatorname{Pr}(A 1.2(v)) \leq \Delta^{-10}:$}

We bound the probability of $A 1.2(v)$ in a similar manner. Let $Y_{2}$ denote the number of colours which appear truly in both $\mathrm{Out}_{v}$ and $N(v) \cap S_{i}$, before the uncolouring in Step 3. Let $Z_{2}$ denote the number of colours which appear on external edges from $v$, and appear truly on $N(v) \cap S_{i}$, before the uncolouring in Step 3. Note that if $Y_{2}+Z_{2} \geq\left|\mathrm{Out}_{v}\right|\left(1+\frac{1}{100}\right)$ and fewer than $\frac{1}{200}\left|\mathrm{Out}_{v}\right|$ vertices in $N(v)$ are uncoloured because of reject degree, then A1.2(v) does not hold.

Because $v \notin H^{\prime},\left|\mathrm{Out}_{v}\right| \leq \frac{1}{10^{4}} \Delta$, and so $\left|N(v) \cap S_{i}\right| \geq\left(1-\frac{1}{10^{4}}\right) \Delta$. By Lemma 2.8(c) and (d), for each $x \in \mathrm{Out}_{v}$, the colour class $x$ lies in has at most $\frac{3}{4} \Delta+\sqrt{\Delta}$ neighbours in $S_{i}$, and by Lemma 2.3, the number of colour classes in $\mathcal{C}_{i}^{\prime}$ of size 2 is at most $10 \epsilon \Delta$. Furthermore, by Lemma 2.8(e), at most $500 \epsilon \Delta$ vertices in $S_{i}$ have external degree at least $\frac{\Delta}{100}$. Therefore, $N(v)$ contains at least $\left|\mathrm{Out}_{v}\right|\left(\left(1-\frac{1}{10^{4}}\right) \Delta-\left(\frac{3}{4} \Delta+\sqrt{\Delta}\right)-10 \epsilon \Delta-500 \epsilon \Delta\right) \geq \frac{\Delta}{5}\left|\mathrm{Out}_{v}\right|$ pairs $x, y$ of strongly non-adjacent vertices, with $x \in \mathrm{Out}_{v}, y \in S_{i}$ forming a colour class of size 1 , and $\left|\mathrm{Out}_{y}\right|<\frac{\Delta}{100}$.

Consider any such pair. By Corollary 2.7, for each dense set $S_{j}, \mid$ Overused $_{j} \mid<$ $\log ^{4} \Delta+1$, and so the probability that $x, y$ receives the same colour is at least $\left(\Delta+1-2\left(\log ^{4} \Delta+1\right) /(\Delta+1)^{2}>\frac{1}{\Delta+2}\right.$ (the worse case is when $x, y$ both belong to ornery sets with disjoint Overused sets). We also require that no other vertices in $N^{+}=$Out $_{v} \cup$ Out $_{y} \cup N(x)$ receive that colour. $\left|N^{+}\right| \leq \frac{\Delta}{10^{4}}+\frac{\Delta}{100}+\Delta$, and for any other dense set $S_{j},\left|N^{+} \cap S_{j}\right| \leq \frac{\Delta}{10^{4}}+\frac{\Delta}{100}+\frac{3}{4} \Delta+\sqrt{\Delta}<\frac{4 \Delta}{5}$. Therefore, the probability that this colour doesn't appear anywhere else in $N^{+}$is at least $\left(\frac{1}{5}\right)^{\left|N^{+}\right| / \frac{\Delta \Delta}{5}}>\frac{1}{10}$. Therefore, $\operatorname{Exp}\left(Y_{2}\right) \geq \frac{1}{50}\left|\mathrm{Out}_{v}\right|$, and it follows as in our analysis of $Z_{1}$ that $\operatorname{Pr}\left(Y_{2} \leq \frac{1}{80}\left|\mathrm{Out}_{v}\right|\right) \leq \mathrm{e}^{-\theta\left(\left|\mathrm{Out}_{v}\right|\right)}$.

Let $Z_{2}^{\prime}$ denote the number of colours appearing on the external edges from $v$, which also are initially assigned to internal neighbours of $v$ during Step 1. By Lemma 2.3, $N(v)$ intersects at least $\Delta-\left|\mathrm{Out}_{v}\right|-10 \epsilon \Delta$ colour classes of $\mathcal{C}_{i}$. It is a straightforward application of the Chernoff Bound to verify that with sufficiently high probability it intersects at least $(1-2 \gamma)(\Delta-$ $\left.\mathrm{Out}_{v}-10 \epsilon \Delta\right)$ classes of $\mathcal{C}_{i}^{\prime}$, and that with probability at least $1-\mathrm{e}^{\theta\left(\left|\mathrm{Out}_{v}\right|\right)}$, $Z_{2}^{\prime} \geq \frac{1}{\Delta} \mid$ Out $_{v} \mid(1-3 \gamma)\left(\Delta-\right.$ Out $\left._{v}-10 \epsilon \Delta\right)$. By Lemma 2.8(e), the number of external edges from $S_{i}$ is at most $5 \epsilon \Delta^{2}$, and it follows that the expected value of the sum of the sizes of the external neighbourhoods of the colour classes on which these repeated colours appear is at most $5 \epsilon \Delta^{2} \times \frac{1}{\Delta} \times\left|\mathrm{Out}_{v}\right|$, since 
$Z_{2}^{\prime} \leq \mid$ Out $_{v} \mid$. A straightforward application of Azuma's Inequality shows that this sum is highly concentrated and in particular that with probability at least $1-\mathrm{e}^{-\theta\left(\left|\mathrm{Out}_{v}\right|\right)}$ this sum is at most $6 \epsilon \Delta\left|\mathrm{Out}_{v}\right|$.

We now must bound the number of these colours which appear temporarily. Again, we can do this using the methods with which we bounded $Z_{1}$. If the average external degree of the colour classes assigned the repeated colours is at most $6 \epsilon \Delta$, then the expected number of these colours which will appear temporarily is at most $7 \epsilon Z_{2}^{\prime}$. We consider choosing the assignment of colours for Step 1 as we did in our analysis of event A1.1. The choice of the colour for any colour class $u \notin \mathcal{C}_{i}$ affects this number by at most $\left|N(u) \cap S_{i}\right|$. It follows that $\operatorname{Pr}\left(Z_{2}<\left(\frac{1}{\Delta}\left|\mathrm{Out}_{v}\right|(1-4 \gamma)\left(\Delta-\left|\mathrm{Out}_{v}\right|-10 \epsilon \Delta\right)(1-8 \epsilon)\right)<\mathrm{e}^{-\theta\left(\left|\mathrm{Out}_{v}\right|\right)}\right.$.

Therefore, with probability at least $1-\mathrm{e}^{-\theta\left(\mid \text { Out }_{v} \mid\right)}$, $Y_{2}+Z_{2} \geq\left(\frac{1}{80}+(1-4 \gamma)(1-8 \epsilon)\left(1-10 \epsilon-\frac{\left|\mathrm{Out}_{v}\right|}{\Delta}\right)\right)\left|\mathrm{Out}_{v}\right|>\frac{101}{100}\left|\mathrm{Out}_{v}\right|$. It only remains to be shown that with sufficiently high probability, few enough of the relevant vertices in $S_{i} \cup$ Out $_{v}$ are uncoloured during Step 3.

By Lemma 4.3 , with probability at least $1-\mathrm{e}^{-\theta\left(\left|\mathrm{Out}_{v}\right|\right)}$, fewer than $\frac{1}{10^{20}} \mid$ Out $_{v} \mid$ colour classes in $\mathcal{C}_{i}$ which receive colours appearing on the external edges from $v$ are uncoloured during Step 3. By Lemma 4.2, with probability at least $1-\mathrm{e}^{-\theta\left(\left|\mathrm{Out}_{v}\right|\right)}$, fewer than $\frac{1}{10^{20}}\left|\mathrm{Out}_{v}\right|$ vertices in Out are uncoloured during Step 3. Lastly, we will bound the number of colour classes in $S_{i}$ which receive colours also received in $\mathrm{Out}_{v}$, and which are uncoloured during Step 3.

To do this, we consider choosing our inital colouring in Step 1 in an unusual manner. We first choose an initial colouring using a single application of COL. Let $\mathcal{Z}$ denote the set of colour classes of $\mathcal{C}_{i}^{\prime}$ which receive the same colour as a vertex in $\mathrm{Out}_{v}$. For each $z \in \mathcal{Z}$, we record the colour, $c(z)$, that $z$ receives. We then recolour all the colour classes in $\mathcal{C}_{i}^{\prime}$ using a second permutation of $U_{i}^{\prime}$. Finally, for each $z \in \mathcal{Z}$, we swap within $S_{i}$ the colour $z$ recieved in this second permutation, with $c(z)$. Note that this generates an initial colouring with the same distribution as an application of COL.

Using the same analysis as that in Lemma 4.2, we can show that with probability at least $1-\mathrm{e}^{-\theta\left(\left|\mathrm{Out}_{v}\right|\right)}$, before the swapping fewer than $\frac{1}{1000}|\mathcal{Z}|$ vertices in $\cup_{z \in \mathcal{Z}} z$ are adjacent via a reject edge to a vertex with reject degree at least $\frac{C_{1}}{2}$. The swap creates at most $4|\mathcal{Z}|$ new reject edges, and so at most $4|\mathcal{Z}| / \frac{C_{1}}{2}=\frac{1}{250}|\mathcal{Z}|$ more vertices in $S_{i}$ will be uncoloured in Step 3 because of the swap. Therefore, with probability at least $1-\mathrm{e}^{-\theta\left(\left|\mathrm{Out}_{v}\right|\right)}$, fewer than $\frac{1}{250}|\mathcal{Z}|+\frac{1}{1000}|\mathcal{Z}| \leq \frac{1}{200} \mid$ Out $_{v} \mid$ colour classes in $S_{i}$ which receive colours also 
received in $\mathrm{Out}_{v}$, are uncoloured during Step 3.

Therefore, $\operatorname{Pr}(A 1.2(v)) \leq \mathrm{e}^{-\theta\left(\left|\mathbf{u t}_{v}\right|\right)} \leq \Delta^{-10}$, as $\left|\mathrm{Out}_{v}\right| \geq \log ^{3} \Delta$.

$\operatorname{Pr}(A 1.3(i, a)) \leq \Delta^{-10}:$

To bound the probability of $A 1.3(i, a)$, we define $Z_{3}$ to be the number of vertices $v \in S_{i}$ with external degree at most $a$, which recieve the same colour as an external neighbour during Step 1. Clearly $\operatorname{Pr}(A 1.3(i, a)) \leq \operatorname{Pr}\left(Z_{3}>\right.$ $2 a)$.

First, we will expose the colours assigned in Step 1 to $G-S_{i}$. Then we expose the choice of $\mathcal{C}_{i}^{\prime}$. Next, we choose the colours for the colour classes of $S_{i}$. To do this, we take a random permutation of $\{1, \ldots, \Delta+1\}-$ Overused $_{i}$ and assign the first $\left|\mathcal{C}_{i}^{\prime}\right|$ colours, in order, to the classes of $\mathcal{C}_{i}^{\prime}$ (which were previously ordered arbitrarily). Each colour class $x \in \mathcal{C}_{i}^{\prime}$, recieves a uniformly random colour from $\{1, \ldots, \Delta+1\}-$ Overused $_{i}$. Therefore $\operatorname{Exp}\left(Z_{3}\right) \leq\left|S_{i}\right| \times$ $a /\left(\Delta+1-\mid\right.$ Overused $\left._{i} \mid\right)<1.5 a$, by Lemmas 2.1 and 2.6 .

Clearly, swapping two colours in the permutation changes $Z_{3}$ by at most 2. Therefore, by Azuma's Inequality (see Remark 1.4), $\operatorname{Pr}\left(Z_{3}>2 a\right) \leq$ $\mathrm{e}^{-\theta(\Delta)}<\Delta^{-10}$.

$\operatorname{Pr}(A 1.4(i, c)) \leq \Delta^{-10}$

To bound the probability of $A 1.4(i, c)$, we define $Z_{4}$ to be the number of vertices $v \in S_{i}$ which have an external neighbour outside of $\mathrm{Big}_{i}$ which receives colour $c$ during Step 1. Clearly $\operatorname{Pr}(A 1.4(i, c)) \leq \operatorname{Pr}\left(Z_{4}>\Delta^{31 / 32}\right)$.

Each vertex in $G$ receives a uniformly random colour from $\{1, \ldots, \Delta+1\}$, or from $\{1, \ldots, \Delta+1\}-$ Overused $_{j}$ in the case that the vertex is in an ornery set $S_{j}$, and so the probability that $v$ receives $c$ is at most $1 /\left(\Delta+1-\right.$ Overused $\left._{j}\right)<$ $\frac{2}{\Delta}$. Therefore, $\operatorname{Exp}\left(Z_{4}\right) \leq \frac{2}{\Delta} \sum_{v \in G-\left(S_{i} \cup \mathrm{Big}_{i}\right)} \operatorname{deg}_{S_{i}}(v) \leq 3 \log ^{7} \Delta$, by Lemma $2.8(\mathrm{f})$.

Changing the colour of any vertex, or flipping the colour of any two vertices in $G-\left(S_{i} \cup \mathrm{Big}_{i}\right)$ affects $Z_{4}$ by at most $2 \Delta^{7 / 8}$. Therefore, by Azuma's Inequality (see Remark 1.4), $\operatorname{Pr}\left(Z_{4}>\Delta^{31 / 32}\right) \leq \mathrm{e}^{-\theta\left(\Delta^{1 / 32} / \log ^{7} \Delta\right)}<\Delta^{-10}$.

$$
\begin{aligned}
& \operatorname{Pr}(A 1.5(i)) \leq \Delta^{-10}: \\
& \quad \operatorname{Pr}(A 1.5(i)) \leq \mathrm{e}^{-\theta(\Delta)}<\Delta^{-10} \text { by Lemma 4.2. }
\end{aligned}
$$




\section{$\operatorname{Pr}(A 1.6(v)) \leq \Delta^{-10}:$}

Finally, we bound the probability of $A 1.6(v)$. For any $v$ in some dense set $S_{i}$, expose the choice of $\mathcal{C}_{i}^{\prime}$. There are at most $(\gamma+10 \epsilon) \Delta<1.5 \gamma \Delta$ edges from $v$ to $\mathcal{C}_{i}-\mathcal{C}_{i}^{\prime}$. Thus, the expected number of colours on these edges which are not in $A_{i}^{\prime}$ is at most $\gamma \times 1.5 \gamma \Delta$. A straightforward application of the Chernoff Bound yields that the probability of more than $2 \gamma^{2} \Delta$ of these colours not being in $A_{i}^{\prime}$ is at most $\mathrm{e}^{-\theta(\Delta)}$. The probability that more than $\frac{1}{10^{19}} \Delta$ other colours will be added to $P R(v)$ is at most the probability that more than $\frac{2}{10^{19}} \Delta$ classes will be uncoloured during Step 3, which by Lemma 4.2 , is at most $\mathrm{e}^{-\theta(\Delta)}$. Therefore, $\operatorname{Pr}(A 1.6(v)) \leq \mathrm{e}^{-\theta(\Delta)}<\Delta^{-10}$.

\section{Phase 2: Finishing the Dense Sets.}

At the beginning of this phase, each $S_{i}$ has a set of uncoloured colour classes, $W_{i}$, and a set of unused colours $U_{i}$. In this phase, we will randomly match the colours of $U_{i}$ to the classes in $W_{i}$.

For each $S_{i}$, we will match the colours of $U_{i}$ to the classes $W_{i}$ via a random permutation. We will not be able to retain some of the colours because of high reject degrees, and so we will have to perform several iterations of this process. We will continue until the number of uncoloured classes is at most $\log ^{3} \Delta$, at which point we will simply assign a temporary colour to each remaining vertex.

Often when a class receives and retains a colour, one, or perhaps two, reject edges will be formed. The other endpoint of each of these edges will become critical. In each successive iteration, no class $w$ may retain a colour appearing on an edge from a vertex of $w$ to a critical vertex. We say such a colour is forbidden for $w$, and we denote by $F(w)$ the set of forbidden colours. Furthermore, if in one iteration, at least $C_{2}$ reject edges incident to one vertex are formed, then none of the corresponding colour classes will retain their colour. Note that this implies that no vertex will have its reject degree increased by more than $C_{2}$ throughout this phase.

For the analysis of this phase, it will be important that any colour class 
$w \in W$ receives and retains each colour in $U-F(w)$ with equal probability. If we only uncolour because of reject degree, then this is not neccessarily true, as a class may be more likely to retain one colour than another. We compensate for this as follows. If a class $w$ receives a colour $c$, and does not lose it because of reject degrees, then we make a final random "flip", i.e. $w$ loses $c$ with probability $\rho(w, c)$, where $\rho(w, c)$ is chosen so that, conditional on $w$ recieving $c, w$ retains $c$ with probability $\rho$, where $\rho$ is a constant independent of $c, w$. We will specify $\rho, \rho(c, w)$ later.

If any vertex receives a colour already assigned to one of its external neighbours or edges, then we consider that vertex to be temporarily coloured. For each uncoloured vertex $v$, we define $T(v)$ to be the set of such colours.

Phase 2 runs as follows:

1. Initialize $W_{i, 1}=W_{i}, U_{i, 1}=U_{i}$, and for each $w \in W_{i}, F_{1}(w)=\emptyset$.

2. For $k=1$ to $k_{0}=\left\lceil\left(3 \log \log \Delta-\log \left|W_{i}\right|-\log 2\right) / \log \tau\right\rceil$ (where $\tau$ is a constant to be specified later):

(a) Initialize $W_{i, k+1}=W_{i, k}, U_{i, k+1}=U_{i, k}$, and for each $w \in W_{i, k}, F^{+}(w)=$ $\emptyset$. (Remark: $F^{+}(w)$ is the set of colours newly forbidden to $w$ during this iteration. It is incremented during the procedure COLOUR.)

(b) We apply the procedure ADJUSTSIZES to modify the set $F_{k}(w)$ for each $w \in W_{i, k}$, yielding new sets $F_{k}^{*}(w)$ where $\left|F_{k}^{*}(w)\right|=$ $\frac{1}{20}\left|W_{i, k}\right|$ for each $w \in W_{i, k}$ and $\left|\left(F_{k}^{*}\right)^{-1}(c)\right|=\frac{1}{20}\left|W_{i, k}\right|$ for each $c \in U_{i, k}$.

(c) Choose a random permutation $\phi: W_{i, k} \rightarrow U_{i, k}$.

(d) For each $w \in W_{i, k}$, if

i. $\phi(w) \notin F_{k}^{*}(w)$ and

ii. there is no neighbour $x \in S_{i}$ of $w$ such that the edge $(x, w)$ is coloured $\phi(w)$, and there are at least $C_{2}-1$ other neighbours of $x: w_{1}, \ldots, w_{C_{2}-1} \in W_{i, k}$ with each edge $\left(x, w_{j}\right)$ coloured $\phi\left(w_{j}\right)$.

then with probability $1-\rho(w, \phi(w)), \operatorname{COLOUR}(w, \phi(w))$.

(e) For each $w \in W_{i, k}, F_{k+1}(w):=\left(F_{k}(w) \cup F^{+}(w)\right) \cap U_{i, k+1}$ 
3. We temporarily colour the classes of $W_{i, k_{0}}$ with the colours of $U_{i, k_{0}}$, thus setting $\mathrm{Temp}_{i}:=\mathrm{Temp}_{i} \cup W_{i, k_{0}}$.

We assign a colour $c$ to a class $w$ through the following procedure:

\section{$\operatorname{COLOUR}(w, c)$}

1. Assign $c$ to $w$.

2. $W_{i, k+1}:=W_{i, k+1}-w, U_{i, k+1}:=U_{i, k+1}-c$.

3. for each vertex $v \in w$, if $c \in T(v)$ then Temp $p_{i}:=\operatorname{Temp}_{i} \cup\{v\}$.

4. for each vertex $v \in w$, if an internal edge $(u, v)$ has colour $c$, then for each edge $\left(u, v^{\prime}\right)$ where $v^{\prime} \in w^{\prime} \in W_{i, k}$, add the colour of $\left(u, v^{\prime}\right)$ to $F^{+}\left(w^{\prime}\right)$.

Before presenting the procedure ADJUSTSIZES, we make an important comment on 2 technical steps. For ease of analysis, we will never allow $F_{k}(w)$ to be larger than $\frac{1}{40}\left|U_{i, k}\right|$, nor will we allow any colour to appear in more than $\frac{1}{40}\left|U_{i, k}\right|$ sets $F_{k}(w)$. If the number of forbidden colours for a class (or the number of classes for which a colour is forbidden) ever exceeds this bound, then we remove some colours from some forbidden sets. The danger here is, of course, that if this happens then there is no guarantee that the reject degrees will be bounded. However, as we will show in Corollary 5.4, with sufficiently high probability this never happens, and so the reject degree of each vertex increases by at most $C_{2}$ during Phase 2 .

\section{ADJUSTSIZES}

1. For each $w \in W_{i, k}$, if $\left|F_{k}(w)\right|>\frac{1}{40}\left|U_{i, k}\right|$ then we replace $F_{k}(w)$ by an arbitrary subset $Q_{1} \subseteq F_{k}(w)$ of size $\frac{1}{40}\left|U_{i, k}\right|$.

2. For each $c \in U_{i, k}$, if $\left|F^{-1}(c)\right|>\frac{1}{40}\left|U_{i, k}\right|$ then we select an arbitrary subset $Q_{2} \subset F_{k}^{-1}(c)$ of size $\frac{1}{40}\left|U_{i, k}\right|$, and delete $c$ from $F_{k}(w)$ for each $w$ in $F_{k}^{-1}(c)-Q_{2}$.

3. We select for each $w \in W_{i, k}$ a superset $F^{*}(w) \supset F(w), F^{*}(w) \subseteq U_{i, k}$, of size $\frac{1}{20}\left|W_{i, k}\right|$ such that $\left|\left(F^{*}\right)^{-1}(c)\right|=\frac{1}{20}\left|W_{i, k}\right|$ for each $c \in U_{i, k}$. 
The only step of Phase 2 which is not straightforward is Step 3 of ADJUSTSIZE. The fact that this step is always possible follows immediately from the following Lemma.

Lemma 5.1 Let $H$ be any bipartite graph on an $n \times n$ bipartition, with maximum degree at most $t \leq \frac{n}{9}$. There exists a 2 -regular bipartite supergraph $H^{\prime} \supset H$ on the same vertex set.

Proof Denote the parts of the bipartition by $A, B$. Consider the following network flow problem defined on the graph $\bar{H}$ with every edge directed from $A$ to $B$. Each $a \in A$ has a supply of $x(a)=2 t-\operatorname{deg}_{H}(a)$, and each $b \in B$ has a demand of $y(b)=2 t-\operatorname{deg}_{H}(b)$. (Note that $\sum_{a \in A} x(a)=$ $\sum_{b \in B} y(b)$.) The capacity of each edge is 1 . Clearly, it suffices to show that this problem has a solution.

If not, then by the Max-flow Min-cut Theorem (see, for example, [7]) there are partitions $A=A_{1} \cup A_{2}, B=B_{1} \cup B_{2}$ such that $\sum_{a \in A_{1}} x(a)-\sum_{b \in B_{1}} y(b)>$ $\left|E_{H}\left(A_{1}, B_{2}\right)\right|$.

Now for each $a, b, t \leq x(a), y(b) \leq 2 t \leq \frac{2 n}{9}$, and $\operatorname{deg}_{\bar{H}}(a), \operatorname{deg}_{\bar{H}}(b) \geq$ $n-t \geq \frac{8 n}{9}$. Thus, if $\left|B_{1}\right| \leq \frac{2 n}{3}$ then $\left|E_{H}\left(A_{1}, B_{2}\right)\right| \geq \frac{2 n}{9}\left|A_{1}\right| \geq \sum_{a \in A_{1}} x(a)$. A similar situation arises if $\left|A_{1}\right| \geq \frac{n}{3}$. Finally, if $\left|A_{1}\right|<\frac{n}{3}$, and $\left|B_{1}\right|>\frac{2 n}{3}$, then $\sum_{a \in A_{1}} x(a)-\sum_{b \in B_{1}} y(b)<0$, and so there is a solution to the network flow problem, and hence $H^{\prime}$ exists.

We also need the following properties to hold for the colouring produced by Phase 2:

(P2.1) For each $i$ and $a \geq \log ^{3} \Delta,\left|\operatorname{Temp}_{i}(a)\right| \leq 5 a$.

(P2.2) For each ornery $S_{i}$, and each colour $c$, the number of vertices in $S_{i}$ having an external neighbour outside of Big $i$ truly coloured $c$ is at most $2 \Delta^{31 / 32}$.

During the $k$ th iteration of the algorithm, for any colour class $w \in W_{k}$, $c \in U_{k}$, we define $R(w, c)$ to be the event that $w$ receives $c$ and there is some neighbour $x \in S_{i}$ of $w$ such that the edge $(x, w)$ is coloured $c$, and there are at least $C_{2}-1$ other neighbours of $x$ : $w_{1}, \ldots, w_{C_{2}-1} \in W_{i, k}$ where each vertex $w_{j}$ receives the colour of $\left(x, w_{j}\right)$. We define $H_{k}$ to be the history of the procedure, i.e. the set of choices made prior to the $k$ th iteration.

Lemma 5.2 For any $k, H_{k}, w, c, \operatorname{Pr}\left(R(w, c) \mid H_{k} \wedge(w\right.$ receives $\left.c)\right) \leq \frac{1}{400}$. 
Proof For any $c$, there are at most 2 choices for $x$. For either choice, let $Z$ be the random variable denoting the number of edges $\left(x, w^{\prime}\right)$, with $w^{\prime} \in W_{i, k}-w$ and where $\phi\left(w^{\prime}\right)$ is the colour appearing on $\left(x, w^{\prime}\right)$.

There are at most $2\left|W_{i, k}\right|$ edges from $x$ to $W_{i, k}$, and each of them becomes a reject edge with probability at most $\left|W_{i, k}\right|^{-1}$. Therefore, $\operatorname{Exp}(Z) \leq 2$, and so by Markov's Inequalilty (see, eg. [9]), $\operatorname{Pr}\left(Z \geq C_{2}-1\right) \leq \frac{\operatorname{Exp}(Z)}{C_{2}-1} \leq \frac{1}{800}$.

Therefore $\operatorname{Pr}\left(R(w, c) \mid H_{k} \wedge(w\right.$ receives $\left.c)\right) \leq \frac{1}{400}$.

We set $\rho=\frac{1}{200}$, and at the beginning of the $k$ th iteration, we define $\rho(w, c)$ to be the unique solution to:

$\operatorname{Pr}\left(R(w, c) \mid H_{k} \wedge(w\right.$ receives $\left.c)\right)+\left(1-\operatorname{Pr}\left(R(w, c) \mid H_{k} \wedge(w\right.\right.$ receives $\left.\left.c)\right)\right) \times \rho(w, c)=\rho$

Note that $\rho(w, c)$ is always non-negative by Lemma 5.2.

Finally, we define $\tau=\frac{1}{20}+\left(1-\frac{1}{20}\right) \rho<\frac{1}{15}$. Note that $\tau$ is both the probability that a particular $w \in W_{k}$ remains in $W_{k+1}$ and the probability that a particular $c \in U_{k}$ remains in $U_{k+1}$.

For each vertex $v \in S_{i}$, we define the potential reject colours, $P R_{k}(v)$, to be the set of colours in $U_{i, k}$ appearing on an edge from $v$ to a vertex in a colour class of $W_{i, k}$, and we set $P R_{k}=\max _{v \in S_{i}}\left\{P R_{k}(v)\right\}$ (recall $P R_{1}(v)=$ $P R(v))$. For each $1 \leq a \leq \Delta$, and $1 \leq k \leq k_{0}$, we denote by $\operatorname{Temp}_{i, k}(a)$ the set of vertices $v$ with $\left|\mathrm{Out}_{v}\right| \leq a$ which in round $k$ receive and retain a colour which is on an external edge or an external neighbour. Note that $\cup_{k} \operatorname{Temp}_{i, k}(a) \subseteq \operatorname{Temp}_{i}(a)$.

Lemma 5.3 With probability at least $1-\Delta^{-\theta(\sqrt{\log \Delta})}$, for every $1 \leq k \leq k_{0}$ we have the following:

(a) ||$W_{i, k+1}|-\tau| W_{i, k}|| \leq \log \Delta^{3 / 4} \sqrt{\left|W_{i, k}\right|}$

(b) for each vertex $v \in S_{i},\left|P R_{k+1}(v)\right| \leq \tau^{2}\left|P R_{k}(v)\right|+\log \Delta^{3 / 4} \sqrt{\left|P R_{k}(v)\right|}$,

(c) for each vertex $v \in \cup_{w \in W_{i, k}} w,\left|T(v) \cap U_{i, k+1}\right| \leq \tau\left|T(v) \cap U_{i, k}\right|+$ $\log \Delta^{3 / 4} \sqrt{\left|T(v) \cap U_{i, k}\right|}$,

(d) for each $a \geq \log ^{3} \Delta$, $\left|\operatorname{Temp}_{i, k+1}(a)\right| \leq \max _{\left|\mathrm{Out}_{v}\right| \leq a}\left|T(v) \cap U_{i, k}\right|+$ $\log \Delta^{3 / 4} \sqrt{{\max \mid \text { Out }_{v} \mid \leq a}\left|T(v) \cap U_{i, k}\right|}$, 
(e) for each $w \in W_{i, k},\left|F_{k+1}(w)\right| \leq \tau\left|F_{k}(w)\right|+2 \tau P R_{k}+\log \Delta_{\sqrt[3]{4}} \sqrt{\left|F_{k}(w)\right|+P R_{k}}$,

(f) for each $c \in U_{i, k},\left|F_{k+1}^{-1}(c)\right| \leq \tau\left|F_{k}^{-1}(c)\right|+2 \tau P R_{k}+\log \Delta^{3 / 4} \sqrt{\left|F_{k}^{-1}(w)\right|+P R_{k}}$.

Proof (a) By the previous remarks, $\operatorname{Exp}\left(\left|W_{i, k+1}\right|\right)=\tau\left|W_{i, k}\right|$. We choose our random permutation $\phi$ by choosing an unassigned colour from $U_{i, k}$ for each $w \in W_{i, k}$ in sequence. Swapping two colours can affect $\left|W_{i, k+1}\right|$ by at most $2 C_{2}$, and so each choice affects $\operatorname{Exp}\left(\left|W_{i, k+1}\right|\right)$ by at most $2 C_{2}$. Therefore by Azuma's Inequality, $\operatorname{Pr}\left(|| W_{i, k+1}\left|-\operatorname{Exp}\left(\left|W_{i, k+1}\right|\right)\right|>\log ^{3 / 4} \Delta \sqrt{\left|W_{i, k}\right|}\right) \leq$ $\mathrm{e}^{-\theta\left(\log ^{3 / 2} \Delta\right)}$. The analysis related to (b) to (f) is similar and we omit it, noting only that for each $w \in W_{i, k}, \operatorname{Exp}\left|F_{k+1}(w)\right| \leq \tau\left|F_{k}(w)\right|+2 \tau P R_{k}$, as the first term counts the expected number of members of $F_{k}(w)$ which remain in $U_{i, k+1}$, and the second term counts (in fact greatly overcounts) the expected number of members from $F^{+} \cap U_{i, k+1}$ (and similarly for (f)).

Each of our at most $6 k_{0}\left|S_{i}\right|$ bad events occurs with probability at most $\mathrm{e}^{-\log ^{3 / 2} \Delta}$, and so the probability that none of them occur is at least $1-$ $\Delta^{-\theta(\sqrt{\log \Delta})}$.

Corollary 5.4 With probability at least $1-\Delta^{-\theta(\sqrt{\log \Delta})}$, for each $0 \leq k \leq k_{0}$,

(a) $\frac{1}{2} \tau^{k}\left|W_{i}\right| \leq\left|W_{i, k}\right| \leq 2 \tau^{k}\left|W_{i}\right|$,

(b) $P R_{k} \leq 6 \tau^{2 k} \gamma\left|W_{i}\right|$,

(c) for each vertex $v \in \cup_{w \in W_{k}} w,\left|T(v) \cap U_{i, k}\right| \leq 4 \tau^{k}\left|\mathrm{Out}_{v}\right|+\log ^{1.75} \Delta$,

(d) for each $w \in W_{i, k},\left|F_{k}(w)\right| \leq \frac{1}{40}\left|W_{i, k}\right|$,

(e) for each $c \in U_{i, k},\left|F_{k}^{-1}(c)\right| \leq \frac{1}{40}\left|W_{i, k}\right|$.

Proof Consider the sequence defined by $x_{0}=\left|W_{i}\right|, x_{k+1}^{+}=\tau x_{k}+$ $x_{k}^{13 / 14}, x_{k+1}^{-}=\tau x_{k}-x_{k}^{13 / 14}$. If Lemma 5.3(a) holds for each $k$, and $\left|W_{i, k^{\prime}}\right|>$ $\log ^{2} \Delta$ for each $k^{\prime}<k$, then $x_{k}^{+} \leq\left|W_{i, k}\right| \leq x_{k}^{-}$. It is straightforward to verify that for $k \leq k_{0}, \frac{1}{2} \tau^{k} x_{0} \leq x_{k} \leq 2 \tau^{k} x_{0}$, and that $\left|W_{i, k_{0}}\right|>\log ^{2} \Delta$, thus proving (a).

A similar argument, applying property (P1.6) and observing that $\left|W_{i}\right| \geq$ $\gamma \Delta$, yields $P R_{k} \leq 2 \tau^{2 k} P R_{0} \leq 2 \tau^{2 k}\left(3 \gamma\left|W_{i}\right|\right)$, thus proving (b). 
(c) follows in the same manner, with the extra $\log ^{1.75} \Delta$ term required since the error term in Lemma 5.3(c) becomes more significant when $\mid T(v) \cap$ $U_{i, k} \mid<\log ^{1.75} \Delta$.

If Lemma 5.3(a,e) and Corollary $5.4(\mathrm{a}, \mathrm{b}, \mathrm{d})$ hold for each $k$, then recalling that $\left|U_{i, k}\right|=\left|W_{i, k}\right|$, we have that for each $w \in W_{i, k}$,

$$
\begin{aligned}
\frac{\left|F_{k+1}(w)\right|}{\left|U_{i, k+1}\right|} & \leq \frac{\tau\left|F_{k}(w)\right|}{\left|U_{i, k+1}\right|}+\frac{12 \tau\left(\tau^{2 k} \gamma\left|U_{i}\right|\right)}{\left|U_{i, k+1}\right|}+\frac{\log \Delta \sqrt{2\left|U_{i, k}\right|}}{\left|U_{i, k+1}\right|} \\
& \leq \frac{\left|F_{k}(w)\right|}{\left|U_{i, k}\right|}\left(1+\frac{\log ^{3 / 4} \Delta}{\sqrt{U_{i, k+1}}}\right)+24 \gamma \tau^{k}+\frac{\log \Delta \sqrt{2\left|U_{i, k}\right|}}{\left|U_{i, k+1}\right|} \\
& \leq \frac{\left|F_{k}(w)\right|}{\left|U_{i, k}\right|}+25 \gamma \tau^{k} .
\end{aligned}
$$

Since $F_{0}(w)=\emptyset$, it follows that

$$
\begin{aligned}
\left|F_{k}(w) \cap U_{i, k}\right| & \leq\left|U_{i, k}\right| \times \sum_{j=0}^{k-1} 25 \gamma(\tau)^{j} \\
& <\frac{1}{50}\left|U_{i, k}\right|
\end{aligned}
$$

The same analysis applies to (e).

Lemma 5.5 For each $S_{i}$, property (P2.1) holds with probability at least 1 $\Delta^{-6}$

Proof If Corollary 5.4 holds for each $k$, then the number of uncoloured colour classes at the end of Step 1 is at most $2 \tau^{k_{0}}\left|W_{i}\right|<\frac{1}{4} \log ^{3} \Delta$. Thus, for any $a \geq \log ^{3} \Delta$, the number of vertices added to $\operatorname{Temp}_{i}(a)$ in Step 2 is at most $\frac{1}{2} a$.

By Lemma 5.3 and Corollary 5.4 , with probability at least $1-\Delta^{-\theta(\sqrt{\log \Delta})}$, the number of vertices added to $\operatorname{Temp}_{i}(a)$ during Step 1 is at most

$$
2 a+\sum_{k=1}^{k_{0}}\left(4 \tau^{k} a+\log ^{1.75} \Delta+\log ^{.75} \Delta \sqrt{a}\right)<2.25 a+\log ^{2.5} \Delta<2.5 a,
$$


and so the lemma follows from (P1.3).

Lemma 5.6 For each $S_{i}$, property (PQ.D) holds with probability at least 1 $\Delta^{-6}$.

Proof The proof is virtually identical to the proof of the bound on $\operatorname{Pr}(A 1.4(i, c))$ in Lemma 4.4, after observing that for each colour $c$, at most one vertex in each dense set receives $c$.

Lemma 5.7 With positive probability, properties (P2.1) and (PQ.2) hold for the colouring produced by Phase 2 , and the reject degree of each vertex increases by at most $C_{2}$.

Proof We define the following events:

A2.1( $i, a)$ - Defined for each $1 \leq i \leq \ell$ and $\log ^{3} \Delta \leq a \leq \Delta$, this is the event that $\left|\operatorname{Temp}_{i}(a)\right|>50 a$.

$A 2.2(i, c)$ - Defined for each $i$ such that $S_{i}$ is ornery and $c \in\{1, \ldots, \Delta\}$, this is the event that the number of vertices in $S_{i}$ having an external neighbour outside of $\mathrm{Big}_{i}$ with colour $c$ is more than $2 \Delta^{31 / 32}$.

A2.3(i) - Defined for each $1 \leq i \leq \ell$, this is the event that for some $1 \leq k \leq k_{0}$ and for some $w \in W_{i}$ or $c \in U_{i},\left|F_{k}(w)\right|$ at some point exceeds $\frac{1}{800}\left|W_{i, k}\right|$ or $\left|F_{k}^{-1}(c)\right|$ at some point exceeds $\frac{1}{800}\left|W_{i, k}\right|$.

If $S_{i}, S_{j}$ are at distance at least 3 from each other, then any two events A2. $x_{1}\left(i, y_{1}\right), A 2 . x_{2}\left(j, y_{2}\right)$ are independent. Thus, each event is independent of all but at most $\Delta^{3}$ other events. Furthermore, by Lemmas 5.5, 5.6, Corollary 5.4 and properties $(\mathrm{P} 1.1)$ and $(\mathrm{P} 1.2)$, the probability of each event is less than $\Delta^{-6}$. Therefore, our lemma follows from the Local Lemma.

\section{Phase 3: The Temporary Colours}

In this phase, we change the colours of all the temporarily coloured vertices, other than those in $H^{\prime}$ which we deal with in Phase 4 . The first step is to 
deal with the kernels of the dense sets. After that, we process the dense sets $S_{1}, \ldots, S_{\ell}$ in sequence. Within each dense set, we recolour the temporarily coloured vertices in nondecreasing order of their external degrees. These vertices will then be considered to be truly coloured.

\subsection{Step 1: The Kernels of the Ornery Sets.}

For each ornery $S_{i}$, we will recolour all vertices in $\operatorname{Temp}_{i} \cap K_{i}$, such that the following properties hold.

(Q3.1) We create no new external reject edges.

(Q3.2) There are no adjacent $v \in K_{i}, u \in S_{j}, j \leq i$, with the same colour.

(Q3.3) The reject degree of any vertex increases by at most 3 .

In order for future steps to succeed, we will also need a few more properties.

(P3.1) For each $v \in H \cup H^{\prime}, N(v)$ has at least $\frac{\epsilon \gamma^{3}}{8} \Delta$ colours which appear truly twice.

(P3.2) For each $v \in G-\left(H \cup H^{\prime}\right)$ such that $\left|\mathrm{Out}_{v}\right| \geq \log ^{3} \Delta$, the number of colours appearing truly twice in $N(v)$ exceeds the number of colours on external edges of $v$ which do not appear truly on $N(v)$ by at least $\frac{1}{300}\left|\mathrm{Out}_{v}\right|$.

For each ornery set $S_{i}$, and each $v \in \operatorname{Temp}_{i} \cap K_{i}$, we will swap the colour of $v$ with that of a vertex $u \in K_{i}-$ Temp $_{i}$. Of course, we must select $u$ carefully. Consider any colour class $w$ of colour $c$ which contains a vertex in Temp $_{i} \cap K_{i}$. We refer to $w$ as a swapping class, and we define Swappable Sw $_{w}$ to be the set of colour classes $w^{\prime} \in \mathcal{C}_{i}$ with the following properties:

(a) $\left|w^{\prime}\right|=1$,

(b) the vertex contained in $w^{\prime}$ lies in $K_{i}$,

(c) the vertex contained in $w^{\prime}$ does not lie in Temp , $_{\text {, }}$

(d) no vertices in $\mathrm{Out}_{w^{\prime}}$, nor any external edges from $w^{\prime}$ have colour $c$, and

(e) no vertices in Out ${ }_{w}$, nor any external edges from $w$ have the colour currently assigned to $w^{\prime}$.

Note that swapping the colour of any one swapping class $w$ with that of some $w^{\prime} \in$ Swappable $_{w}$ will preserve properties (Q3.1), (Q3.2) and (Q3.3). However, the cumulative effect of several such swaps might violate (Q3.2) 
and (Q3.3). We will deal with that concern later. First, we will establish

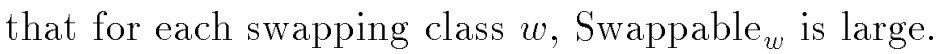

Lemma 6.1 For each swapping class $w$, $\mid$ Swappable $_{w} \mid \geq \frac{\Delta}{\log ^{12} \Delta}$.

Proof We denote the colour of $w$ by $c$.

Let $T_{i}$ be the set of colours appearing on Temp $\cap \cap K_{i}$. Temp Tem $_{i} \cap K_{i}=$ $\operatorname{Temp}_{i}\left(\log ^{6} \Delta\right)$, and so by Property (P2.1), $\mid$ Temp $_{i} \cap K_{i} \mid<5 \log ^{6} \Delta$. Furthermore, recall that $T_{i} \cap$ Overused $_{i}=\emptyset$.

Claim 1: There are at least $\frac{\Delta}{2 \log ^{10} \Delta}$ truly coloured vertices in $K_{i}$ which are neither incident to any external edge of colour $c$, nor adjacent to any vertex of $G-S_{i}$ of colour $c$.

Proof:

Case $1 c$ appears on at least $\frac{\Delta}{\log \Delta}$ edges of $E\left(S_{i}, G-S_{i}\right)$.

Since $c \notin$ Overused $_{i}, c \in$ Moderate $_{i}$, and so by Modification 2 of Section 2.3, no vertex of $\mathrm{Big}_{i}$ has colour $c$. Thus, by Property (P2.2), at most $2 \Delta^{31 / 32}$ vertices of $K_{i}$ are adjacent to a node of colour $c$. Also, because $c \notin$ Overused $_{i}$, at most $\Delta-\frac{\Delta}{\log ^{10} \Delta}$ nodes of $K_{i}$ are incident to an edge of $E\left(S_{i}, G-S_{i}\right)$ of colour $c$. By Lemma $2.5(\mathrm{~b})$ and the fact that $S_{i}$ is ornery, $\left|K_{i}\right|>\Delta-\log ^{4} \Delta-\log ^{5} \Delta$, and by Property $\mathrm{P}(2.1)$ all but at most $5 \log ^{6} \Delta$ of its elements are truly coloured. Thus we have at least $\Delta-\log ^{4} \Delta-\log ^{5} \Delta-$ $5 \log ^{6} \Delta-2 \Delta^{31 / 32}-\left(\Delta-\frac{\Delta}{\log ^{10} \Delta}\right)>\frac{\Delta}{2 \log ^{10} \Delta}$ truly coloured vertices which are neither incident to any edge of $E\left(S_{i}, G-S_{i}\right)$ nor any vertex of $G-v$ of colour c.

Case $2 c$ appears on fewer than $\frac{\Delta}{\log \Delta}$ edges of $E\left(S_{i}, G-S_{i}\right)$.

At most one colour class within $\mathrm{Big}_{i}$ has colour $c$, and it has less than $\frac{3 \Delta}{4}$ neighbours in $K_{i}$. The rest follows as in Case 1 .

Since $S_{i}$ is ornery, $\left|\mathcal{C}_{i}\right| \geq \Delta-\log ^{4} \Delta$, and by Lemma $2.5,\left|S_{i}\right| \leq \Delta+\log ^{5} \Delta$. Thus, the number of vertices in $S_{i}$ which do not lie in singleton colour classes is at most $3 \log ^{5} \Delta$. Since $w \in K_{i},\left|\mathrm{Out}_{w}\right| \leq \log ^{6} \Delta$ and so at most $2 \log ^{6} \Delta$ colour classes of $\mathcal{C}_{i}$ have a colour appearing on an external neighbour of $w$ or an external edge from $w$. Also, again by Property (P2.1), $\left|\operatorname{Temp}_{i} \cap K_{i}\right| \leq$ $5 \log ^{6} \Delta$.

Therefore, by Claim 1, $\mid$ Swappable ${ }_{w} \mid \geq \frac{\Delta}{2 \log ^{10} \Delta}-3 \log ^{5} \Delta-2 \log ^{6} \Delta-$ $5 \log ^{6} \Delta \geq \frac{\Delta}{\log ^{11} \Delta}$. 
Consider any swapping class $w$ of colour $c$. We will select a subset Candidate $_{w} \subseteq$ Swappable $_{w}$ of size 20, whose elements we refer to as candidates. We will require the following condition:

(Q3.4) There is some $w^{\prime} \in$ Candidate $_{w}$ of colour $c^{\prime}$ such that

(a) $w^{\prime}$ is not a candidate for any other swapping class,

(b) $w^{\prime}$ is not an external neighbour of any member of Candidate $x$ for any swapping class $x$ (in some other dense set), which also has colour $c$.

(c) $w^{\prime}$ is not an external neighbour of any swapping class $x$, which has a candidate of colour $c$,

(d) no candidate of any swapping class which has an external neighbour in $w$ has colour $c^{\prime}$, and

(e) there is no vertex $u$, swapping class $w_{1}$ of colour $c_{1}$, and candidate $w_{1}^{\prime}$ of $w_{1}$ with colour $c_{1}^{\prime}$, all in the same dense set as $w$ and $w^{\prime}$, and with

i. the edge $\left(u, w^{\prime}\right)$ present and coloured $c$, and the edge $\left(u, w_{1}^{\prime}\right)$ present and coloured $c_{1}$,

ii. the edge $\left(u, w^{\prime}\right)$ present and coloured $c$, and an edge from $u$ to $w_{1}$ present and coloured $c_{1}^{\prime}$,

iii. an edge from $u$ to $w$ present and coloured $c^{\prime}$, and the edge $\left(u, w_{1}^{\prime}\right)$ present and coloured $c_{1}$, or

iv. an edge from $u$ to $w^{\prime}$ present and coloured $c^{\prime}$, and an edge from $u$ to $w_{1}^{\prime}$ present and coloured $c_{1}^{\prime}$.

For each swapping class $w$, we will swap the colour on $w$ with that on the class referred to in (Q3.4). (Q3.4) enforces properties (Q3.1), (Q3.2) and (Q3.3). We will require another condition on our candidates, to enforce properties (P3.1) and (P3.2).

To enforce (P3.1), it suffices to bound the number of vertices in the neighbourhood of any $v \in H \cup H^{\prime}$ which are candidates of any swapping class. To enforce (P3.2), we make the following definitions. Consider any vertex $v \in G-\left(H \cup H^{\prime}\right)$ with $\left|\mathrm{Out}_{v}\right| \geq \log ^{3} \Delta$. Let $C_{1}(v)$ be the set of colours which each appear truly at least once in $\mathrm{Out}_{v}$, and truly at least once in $N(v) \cap S_{i}$. Let $D_{1}(v)$ be the set of vertices in $N(v)$ which are truly coloured with colours 
from $C_{1}(v)$. Let $D_{2}(v)$ be the set of vertices in $N(v)$ which are truly coloured with a colour appearing on some external edge from $v$. To enforce (P3.2) it suffices to bound the number of candidates in $D(v)=D_{1}(v) \cup D_{2}(v)$ for each such $v$. Thus, we require the following condition:

(Q3.5)

(a) for each vertex $v \in H \cup H^{\prime}$, the number of candidates in $N(v)$ is at most $\log ^{20} \Delta$, and

(b) for each vertex $v \in G-\left(H \cup H^{\prime}\right)$ with $\left|\mathrm{Out}_{v}\right| \geq \log ^{3} \Delta$, the number of candidates in $D(v)$ is at most $\frac{1}{\log \Delta}\left|\mathrm{Out}_{v}\right|$.

Note that if (Q3.5) holds, then by (P1.1) and (P1.3), (P3.1) and (P3.2) will both hold after completing all swaps. It only remains to be shown that we can in fact construct the sets Candidate . $^{\text {. }}$

Lemma 6.2 We can choose the sets Candidate for all swappable classes w, such that (Q3.4) and (Q3.5) hold.

Proof To choose Candidate $w$, we simply select 20 members of Swappable $w$ at random. We will use the Local Lemma to prove that (Q3.4) and (Q3.5) hold for every swapping class $w$ with positive probability.

For each swapping class $w$, we define $A 3.4(w)$ to be the event that (Q3.4) fails to hold for $w$, and for each vertex $v$, we define $A 3.5(v)$ to be the event that (Q3.5) fails to hold for $v$. It is straightforward to verify that for any swapping class $w, A 3.4(w)$ is independent of the set $\left\{A 3.4\left(w_{1}\right): \operatorname{dist}\left(w, w_{1}\right) \geq\right.$ $6\} \cup\{A 3.5(v): \operatorname{dist}(w, v) \geq 6\}$, and similarly for $A 3.5(v)$ for each $v$. Thus, it suffices to show that both $\operatorname{Pr}(A 3.4(w))$ and $\operatorname{Pr}(A 3.5(v))$ are less than $\Delta^{-8}$.

First we will bound the probability of $A 3.5(v)$. Consider any vertex $v$. Let $N_{1}(v)$ be the union over all ornery $S_{i}$ such that $\left|N(v) \cap S_{i}\right| \geq \frac{\Delta}{2 \log ^{11} \Delta}$ of $N(v) \cap S_{i}$, and let $N_{2}(v)$ be the union over all other ornery $S_{i}$ of $N(v) \cap S_{i}$.

$N_{1}(v)$ meets at most $2 \log ^{11} \Delta$ ornery sets, and each contains at most $5 \log ^{6} \Delta \times 20$ candidates. Therefore, $N_{1}(v)$ contains at most $200 \log ^{17} \Delta$ candidates.

To bound the number of candidates in $N_{2}(v)$, note that for any vertex $u \in N_{2}(v)$, and any $N^{\prime} \subseteq N_{2}(v)-\{u\}$, conditional on any partition of $N^{\prime}$ into candidates and non-candidates, the probability that $u$ is a candidate is at most $5 \log ^{6} \Delta \times\left(20 / \frac{\Delta}{2 \log ^{11} \Delta}\right) \leq \frac{\log ^{18} \Delta}{\Delta}$. Thus, the probability that the number 
of candidates in $N_{2}(v)$ exceeds $\frac{1}{2} \log ^{19} \Delta$ is at most the probability that the binomial variable $\operatorname{BIN}\left(\left|N_{2}(v)\right|, \frac{\log ^{18} \Delta}{\Delta}\right)$ exceeds $\frac{1}{2} \log ^{19} \Delta$ which is much less than $\Delta^{-8}$. Therefore, the probability that $N(v)$ contains more than $\log ^{19} \Delta$ candidates is less than $\Delta^{-8}$.

The same analysis applies to the number of candidates in $\mathrm{Out}_{v}$, and also to the number of candidates in $D(v)$, by observing that since no colour appears more than twice in $S_{i}$, we have $|D(v)| \leq 5 \mid$ Out $_{v} \mid$, and so $\operatorname{Pr}(A 3.5(v)) \leq \Delta^{-8}$.

To bound the probability of $A 3.4(w)$ for any swappable vertex $w$, we will first select the candidates of all swapping vertices other than $w$, and then we will select the candidates for $w$.

Suppose $w \in K_{i}$ and $w$ has colour $c$. Before we select the candidates for $w$, we will consider the number of members of Swappable $w$ which would make bad candidates, i.e. those that would not meet the criteria of condition (Q3.4). They fall into the following five subsets of Swappable ${ }_{w}$.

$\operatorname{Bad}_{1}=\left\{w^{\prime} \mid w^{\prime}\right.$ is a candidate for another swapping class $\left.w_{1}\right\}$

$\operatorname{Bad}_{2}=\left\{w^{\prime} \mid w^{\prime}\right.$ is an external neighbour of a candidate for another swapping class $w_{1}$, of colour $\left.c\right\}$

$\operatorname{Bad}_{3}=\left\{w^{\prime} \mid w^{\prime}\right.$ is an external neighbour of another swapping class $w_{1}$, which has a candidate of colour $c\}$

$\operatorname{Bad}_{4}=\left\{w^{\prime} \mid w^{\prime}\right.$ has the same colour as a candidate for another swapping class $w_{1}$, which has an external neighbour in $\left.w\right\}$

$\operatorname{Bad}_{5}=\left\{w^{\prime} \mid\right.$ there is some vertex $u$, and another swapping class $w_{1}$ of colour $c_{1}$ and with candidate $w_{1}^{\prime}$, violating condition $\left.(\mathrm{Q} 3.4)(\mathrm{e})\right\}$

We set $\mathrm{Bad}=\cup_{i=1}^{5} \operatorname{Bad}_{i} . \quad A 3.4(w)$ is the event that each of the 20

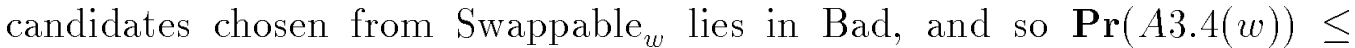
$\left(|\mathrm{Bad}| / \mid \text { Swappable }_{w} \mid\right)^{20}$. We will show that with probability at least $1-\Delta^{-9}$, $|\mathrm{Bad}| \leq \log ^{40} \Delta$, and so, conditional on this bound holding,

$$
\operatorname{Pr}(A 3.4(w)) \leq\left(\frac{\log ^{40} \Delta}{\frac{\Delta}{\log ^{11} \Delta}}\right)^{20} \leq \Delta^{-9}
$$

Thus, $\operatorname{Pr}(A 3.4(w)) \leq 2 \Delta^{-9} \leq \Delta^{-8}$.

It only remains to bound the size of Bad.

Since there are at most $5 \log ^{6} \Delta$ swapping classes in $S_{i}$, each having 20 candidates, we have $\left|\operatorname{Bad}_{1}\right| \leq 100 \log ^{6} \Delta$.

To bound the size of $\mathrm{Bad}_{2}$, we recall that by Lemma 2.5, there are at most $\Delta \log ^{7} \Delta$ external edges from $S_{i}$. Thus, we can apply similar analysis 
to that used to bound $\operatorname{Pr}(A 3.5(v))$ to show that with probability at least $1-\Delta^{-9}, S_{i}$ has at most $\log ^{27} \Delta$ neighbours which are candidates. Because each such candidate lies in the kernel of another ornery set, it has at most $\log ^{6} \Delta$ neighbours in $S_{i}$, and so we have $\operatorname{Pr}\left(\left|\operatorname{Bad}_{2}\right|>\log ^{33} \Delta\right) \leq \Delta^{-9}$.

To bound the size of $\mathrm{Bad}_{3}$, we note that by Lemma 2.5, there are at most $\Delta \log ^{7} \Delta$ swapping classes in other ornery sets which have external neighbours in $S_{i}$. Each of these picks a candidate of colour $c$ with probability at most $20 / \frac{\Delta}{\log ^{11} \Delta}$, and these choices are independent. Thus, $\operatorname{Pr}\left(\left|\operatorname{Bad}_{3}\right|>\log ^{30} \Delta\right) \leq$ $\operatorname{Pr}\left(B I N\left(\Delta \log ^{7} \Delta, 20 \log ^{11} \Delta / \Delta\right)>\log ^{20} \Delta\right) \leq \Delta^{-10}$.

Since $w$ has at most $\log ^{7} \Delta$ external neighbours, $\left|\operatorname{Bad}_{4}\right| \leq 100 \log ^{7} \Delta$.

Since $S_{i}$ has at most $100 \log ^{6} \Delta$ candidates, $\left|\operatorname{Bad}_{5}\right| \leq 400 \log ^{6} \Delta$.

Therefore, with probability at least $1-2 \Delta^{-10}>1-\Delta^{-9}$, $|\mathrm{Bad}| \geq$ $100 \log ^{5} \Delta+\log ^{33} \Delta+\log ^{30} \Delta+100 \log ^{7} \Delta+400 \log ^{6} \Delta<\log ^{40} \Delta$, and the result follows.

\subsection{Step 2: The Remaining Temporary Colours.}

At this point, we will remove the artificial edges and vertices added to $G$ in Section 2.3, as they were needed only to enable us to recolour the temporary vertices in the kernels of the ornery sets in Section 3.1. From this point on, it is important to note that the maximum degree in $G$ has returned to $\Delta$.

The remaining temporarily coloured vertices are relatively straightforward to deal with. We wish to colour all such vertices in $G-H^{\prime}$ (i.e. those with external degree at most $\frac{1}{10^{4}} \Delta$ ) such that (i) we create no new external reject edges, (ii) there are no adjacent $v \in S_{i}, u \in S_{j}, j<i$, with the same colour, and (iii) the reject degree of any vertex increases by at most $C_{3}-3$.

We deal with the sets $S_{i}$ in sequence.

For any $S_{i}$, we first recolour all the temporary vertices with external degree at most $\log ^{3} \Delta$. If there are any such vertices, then $S_{i}$ is not ornery, for otherwise they would lie in the kernel $K_{i}$, and would already be recoloured. Furthermore, by Property $(\mathrm{P} 2.1),\left|\operatorname{Temp}_{i}\left(\log ^{3} \Delta\right)\right| \leq 5 \log ^{3} \Delta$.

Because $S_{i}$ is not ornery, it has at most $\Delta-\log ^{4} \Delta$ colour classes, and so there are at least $\log ^{4} \Delta$ colours not already used on the vertices of $S_{i}$. We will use these new colours to recolour $\operatorname{Temp}_{i}\left(\log ^{3} \Delta\right)$ in a greedy manner. 
We recolour the vertices one at a time. At each turn, the vertex, $v$, has at most

(a) $5 \log ^{3} \Delta$ new colours forbidden because they have already been used in $S_{i}$,

(b) $2 \log ^{3} \Delta$ new colours forbidden because they appear on an external neighbour, or an external edge of $v$, and

(c) $5 \log ^{3} \Delta /\left(C_{3}-3\right)$ new colours forbidden because of vertices in $S_{i}$ whose reject degrees have already been increased by $C_{3}-3$ during Step 2.

Since we have $\log ^{4} \Delta$ colours to choose from, we will always be successful.

We then recolour the rest of Temp $\mathrm{p}_{i}$ in non-decreasing order of external degree. At each turn, vertex $v$ with external degree $a$ will, by Property (P3.2), have at least $\frac{1}{300} a$ colours available which do not appear on any neighbours of $v$, or any external edges of $v$. By Property (P2.1), at most $5 a$ members of Temp $\mathrm{p}_{i}$ have been coloured thus far, and so there are at most $5 a /\left(C_{3}-3\right)<\frac{1}{300} a$ colours forbidden because of vertices in $S_{i}$ whose reject degrees have already been increased by $C_{3}-3$ during Step 2. Thus, we will always be successful.

\section{$7 \quad$ Phase 4 - Finishing the Sparse Vertices}

At this point, we have a partial proper colouring of $G$, such that:

(a) every vertex in $G-\left(H \cup H^{\prime}\right)$ is coloured,

(b) the reject degree of each vertex is at most $C_{1}+C_{2}+C_{3}$, and

(c) for each uncoloured $v, N(v)$ has at least $\frac{\epsilon \gamma^{3}}{8} \Delta$ colours which appear truly twice (i.e. $\mathrm{P}(3.1)$ holds).

In this, the final stage, we take advantage of (P3.1) to complete our colouring without increasing the reject degree of any vertex by more than $C_{4}$. 


\subsection{Step 1: Almost All the Rest.}

We colour nearly all of the remaining vertices through the following procedure. At the beginning of each iteration, vertices which do not have colours are referred to as colourless. Each colourless vertex receives a colour. Some of these vertices retain their colours, while others are uncoloured. Any colourless vertex which retains its colour throughout that iteration is then referred to as coloured. Such a vertex will never be uncoloured.

Complete $k_{1}=\left\lceil 3 \log \Delta /\left(-\log \left(1-10^{-22}\right)\right)\right\rceil$ iterations of the following:

1. Assign to every colourless vertex, a colour chosen uniformly at random from $\{1, \ldots, \Delta+1\}$.

2. For each $u \in V(G)$ and colourless $x_{1}, \ldots, x_{C_{4} / 2} \in N(u)$ such that each $x_{i}$ receives the colour on the edge $\left(u, x_{i}\right)$ in this iteration, uncolour $x_{1}, \ldots, x_{C_{4} / 2}$.

3. If any vertex, $v$, receives a colour which

(a) appears on a coloured neighbour,

(b) is assigned in this iteration to a colourless neighbour, or

(c) is an element of Crit $(v)$ (defined in Step 4).

then uncolour $v$.

4. If any vertex, $u$, is incident to at least $\frac{C_{4}}{2}$ reject edges formed during Phase 4, then that vertex is said to be critical, and for each uncoloured $v \in N(u)$, if $|\operatorname{Crit}(v)| \leq \frac{1}{10^{30}} \Delta$, then we add the colour of the edge $(u, v)$ to $\operatorname{Crit}(v)$.

Remark 7.1 If for every vertex $v$, the number of critical neighbours of $v$ never exceeds $\frac{1}{10^{30}} \Delta$, then no vertex will have its reject degree increased by more than $C_{4}$. It is this case in which we are most interested, and we justify focussing on this case with Lemma 7.2.

We will also require the following property to hold at the end of Step 1.

(Q4.1) No vertex has more than $\sqrt{\Delta}$ uncoloured vertices within a distance of 2 . 
Lemma 7.2 Consider any round $i \geq 1$, and any two disjoint sets $X, Y$ of colourless vertices, with $|X|,|Y| \leq \frac{1}{10^{20}} \Delta$. Let $\mathcal{H}_{i-1}$ denote the partial colouring produced through the first $i-1$ iterations of Phase 4, and let $\operatorname{Col}_{i}(X)$ denote the vector of colours received by the vertices of $X$ during the ith iteration. For any possible $\mathcal{H}_{i-1}, \operatorname{Col}_{i}(X)$, the probability that no vertex in $Y$ retains its colour during round $i$, conditioned on $\mathcal{H}_{i-1}, \operatorname{Col}_{i}(X)$ and the event that every vertex in $X$ retains its colour, is at most $2(1-\zeta)^{|Y|}$, where $\zeta=\frac{1}{10^{22}}$.

Proof Throughout this proof, all probabilities will be conditional on $\mathcal{H}_{i-1}, \operatorname{Col}_{i}(X)$ and the event that every vertex in $X$ retains its colour. We make reference to colours received and retained during round $i$.

Claim: For any $v \notin X$, any colour $c$, any colourless vertices $v_{1}, \ldots, v_{t}$ and any list of colours $c_{1}, \ldots, c_{t}$, the probability that $v$ receives $c$, conditioned on the event that $v_{k}$ receives $c_{k}$ for each $1 \leq k \leq t$, is at most $\frac{2}{\Delta}$. Furthermore, for each $v$, this probability is at least $\frac{1}{\Delta+1}$ for all but at most $2|X|$ values of $c$.

Proof: We outline the main ideas behind the proof here. A detailed proof is straightforward, but somewhat tedious, and we omit the details.

The conditioning on $\mathcal{H}_{i-1}$ has no effect. Suppose $X=\left\{x_{1}, \ldots, x_{|X|}\right\}$. For each $j=1, \ldots,|X|$, the colour that $x_{j}$ receives exposes at most one reject edge $\left(x_{j}, u_{j}\right)$, and if $v \in N\left(u_{j}\right)$ then conditioning on the event that $x_{i}$ retains that colour somewhat decreases the probability that $v$ receives the colour of the edge $\left(u_{j}, v\right)$, by an amount that is determined in part by $v_{1}, \ldots, v_{k}$ and $c_{1}, \ldots, c_{k}$. Furthermore, if $v \in N\left(x_{i}\right)$ then conditioning on the event that $x_{i}$ retains its colour exposes that $v$ does not receive the same colour as $x_{i}$. Each of the at most $2|X|$ aforementioned colours are assigned to $v$ with a probability that is less than $\frac{1}{\Delta+1}$, perhaps as low as 0 . Each of the remaining colours is equally likely to be assigned to $v$, and so is selected with probability at least $\frac{1}{\Delta+1}$ and at most $1 /(\Delta+1-2|X|)<2 / \Delta$.

Let $E_{1}$ be the event that at least $\left(1-\frac{\epsilon \gamma^{3}}{32}\right)|Y|$ members of $Y$ are uncoloured during Step 3 , and let $E_{2}$ be the event that at least $\left(\frac{\epsilon \gamma^{3}}{32}\right)|Y|$ members of $Y$ are uncoloured during Step 2. We will show that each of these events holds with small probability.

To bound the probability of $E_{1}$, we first expose the colours assigned to all uncoloured vertices outside of $Y$, and then expose the colours assigned to 
the vertices of $Y$, one at a time. For each vertex $v$, we say that a colour is forbidden if it lies in Crit $(v)$ or if it appears on a coloured vertex in $N(v)$. We denote $A_{1}$ to be the number of vertices in $Y$ which receive either a forbidden colour or the same colour as a colourless neighbour outside of $Y, A_{2}$ to be the number of vertices in $Y$ which receive a colour which has already been exposed on a previous member of $Y$, and $A_{3}$ to be the number of vertices which receive a colour which is not forbidden, and is not yet exposed as being assigned to a neighbour or a previous member of $Y$. Note that every vertex counted in $A_{2}$ can cause at most one vertex counted in $A_{3}$ to lose its colour, and so if $E_{1}$ holds, then $A_{2} \geq A_{3}-\left(\frac{\epsilon \gamma^{3}}{32}\right)|Y|$. Therefore $\operatorname{Pr}\left(E_{1}\right) \leq \operatorname{Pr}\left(A_{2} \geq \frac{\epsilon \gamma^{2}}{32}|Y|\right)+\operatorname{Pr}\left(A_{3} \leq \frac{\epsilon \gamma^{2}}{16}|Y|\right)$.

Every time we expose the colour assigned to a vertex in $Y$, the probability that it is one which is already exposed on a previous member of $Y$ is, by our Claim, at most $\frac{2|Y|}{\Delta}$, and the probability that it is neither forbidden, nor already exposed on a neighbour or another member of $Y$ is by Property (P3.1) and our Claim, at least $\frac{1}{\Delta+1}\left(\frac{\epsilon \gamma^{3}}{8} \Delta-|Y|-\frac{1}{10^{30}} \Delta-2|Y|\right)>\frac{\epsilon \gamma^{3}}{12}$. Therefore,

$$
\begin{aligned}
\operatorname{Pr}\left(A_{3} \leq \frac{\epsilon \gamma^{2}}{16}|Y|\right) & \leq \operatorname{Pr}\left(B I N\left(|Y|, \frac{\epsilon \gamma^{2}}{12}\right) \leq \frac{\epsilon \gamma^{2}}{16}|Y|\right) \\
\leq & (1-3 \zeta)^{|Y|}
\end{aligned}
$$

by the Chernoff Bound. Similarly, since $\frac{2|Y|}{\Delta}<\frac{\epsilon \gamma^{3}}{50}$, we have

$$
\begin{aligned}
\operatorname{Pr}\left(A_{2} \geq \frac{\epsilon \gamma^{2}}{32}|Y|\right) & \leq \operatorname{Pr}\left(B I N\left(|Y|, \frac{\epsilon \gamma^{3}}{50}\right) \geq \frac{\epsilon \gamma^{2}}{32}|Y|\right) \\
& \leq(1-3 \zeta)^{|Y|}
\end{aligned}
$$

If $|Y| \geq \frac{1}{2} \sqrt{\Delta}$ then $\operatorname{Pr}\left(E_{1}\right) \leq 2(1-3 \zeta)^{|Y|} \leq(1-2 \zeta)^{|Y|}$.

If $\zeta^{-\frac{2}{\zeta}}<|Y|<\frac{1}{2} \sqrt{\Delta}$ then $B I N\left(|Y|, \frac{2|Y|}{\Delta}\right)$ is asymptotic in distribution to a Poisson variable with mean less than 1 , and so $\operatorname{Pr}\left(A_{2} \geq \frac{\epsilon \gamma^{2}}{32}|Y|\right)<$ $\mathrm{e} /\left(\frac{\epsilon \gamma^{2}}{32}|Y|\right) !<\zeta^{|Y|}$. If $|Y| \leq \zeta^{-\frac{2}{\zeta}}$ then $\operatorname{Pr}\left(A_{2} \geq 1\right)<\mathrm{O}\left(\Delta^{-1}\right)<\zeta^{|Y|}$. Therefore again we have $\operatorname{Pr}\left(E_{1}\right) \leq(1-3 \zeta)^{|Y|}+\zeta^{|Y|} \leq(1-2 \zeta)^{|Y|}$.

To bound $\operatorname{Pr}\left(E_{2}\right)$, we first note that our claim implies that that for any colourless vertices $v_{1}, \ldots, v_{t}$ and any list of colours $c_{1}, \ldots, c_{t}$, the probability that $v_{i}$ receives $c_{i}$ for each $1 \leq i \leq t$ is at most $\left(\frac{2}{\Delta}\right)^{t}$. Using this in place of 
Lemma 4.1, observing that $\frac{\epsilon \gamma^{3}}{32}>10^{-20}$ and following the same proof as that of Lemma 4.2, we can show that $\operatorname{Pr}\left(E_{2}\right)<\left(\zeta^{10^{20}}\right)^{10^{-20}|Y|}=\zeta^{|Y|}$. We omit the details, noting only that the extremely small value of this probability is due to the large value of $C_{4}$ !.

Therefore, the conditional probability that no vertex in $Y$ retains its colour is at most $(1-2 \zeta)^{|Y|}+\zeta^{|Y|}<(1-\zeta)^{|Y|}$

Lemma 7.3 For each vertex $v$, the probability that more than $\frac{1}{10^{30}} \Delta$ neighbours of $v$ become critical is at most $\Delta^{-\sqrt{\Delta}}$.

Given any $U=\left\{u_{1}, \ldots, u_{10^{-30} \Delta}\right\} \subset N(v)$, and any $T_{j}=\left\{t_{j, 1}, \ldots, t_{j, \frac{C_{4}}{2}}\right\} \subset$ $N\left(u_{j}\right), j=1, \ldots, 10^{-30} \Delta$, we consider the probability that for each $j, k, t_{j, k}$ receives and retains $c_{j, k}$, the colour of the edge $\left(t_{j, k}, u_{j}\right)$. Note that each vertex can contribute to the reject degree of at most one other vertex, and so we can assume that the sets $T_{j}$ are disjoint.

For each $t_{j, k}$ we choose an iteration $i_{j, k}$, and we will bound the probability that every $t_{j, k}$ receives and retains $c_{j, k}$ during iteration $i_{j, k}$.

For each iteration $i$, we define $I_{i}=\left\{t_{j, k}: i_{j, k}=i\right\}$. During iteration $i$, the probability that each $t_{j, k} \in I_{i}$ receives and retains $c_{j, k}$ is at most the probability that each $t_{j, k}$ receives $c_{j, k}$ which is $\left(\frac{1}{\Delta+1}\right)^{\left|I_{i}\right|}$. By Lemma 7.2, conditional on this happenning, the probability that each $t \in \cup_{i^{\prime}>i} I_{i^{\prime}}$ is uncoloured during round $i$ is at most $(1-\zeta)^{\left|\cup_{i^{\prime}>i} I_{i^{\prime}}\right|}$.

Therefore, the probability that each vertex in $U$ becomes critical is at most

$$
\begin{aligned}
\left(\begin{array}{c}
\Delta \\
\frac{C_{4}}{2}
\end{array}\right)^{10^{-30} \Delta}\left(\frac{1}{\Delta}\right)^{\frac{C_{4}}{2} 10^{-30} \Delta} \times \sum_{1}(1-\zeta)^{\sum_{j, k}\left(i_{j, k}-1\right)} & \leq\left(\frac{C_{4}}{2} !\right)^{-10^{-30} \Delta} \times\left(\frac{1}{\zeta}\right)^{\frac{C_{4}}{2} 10^{-30} \Delta} \\
& \leq\left(\frac{C_{4}}{100} !\right)^{-10^{-30} \Delta}
\end{aligned}
$$

where the $\operatorname{sum} \sum_{1}$ is over all choices for the set of iterations $i_{j, k}$. Therefore,

$$
\operatorname{Pr}\left(A_{v}\right) \leq\left(\begin{array}{c}
\Delta \\
10^{-30} \Delta
\end{array}\right)\left(\frac{C_{4}}{100} !\right)^{-10^{-30} \Delta}<\mathrm{e}^{-\theta(\Delta)}<\Delta^{-\sqrt{\Delta}}
$$


since $\left(C_{4} / 100\right) !>10^{31}$.

Remark 7.4 The reason that we chose such a high value for $C_{4}$, is that in our proof of Lemma 7.3 it is important that $C_{4}$ be at least as high as a function of $\zeta$. By complicating our argument somewhat further, it is possible to use a much smaller value of $C_{4}$, which is not a function of $\zeta$.

Lemma 7.5 With positive probability, (Q4.1) holds, and the reject degree of each vertex increases by at most $C_{4}-1$.

Proof For each vertex $v$, define $A 4.1(v)$ to be the event that there are more than $\sqrt{\Delta}$ uncoloured vertices of distance at most 2 from $v$, and define $A 4.2(v)$ to be the event that the number of critical neighbours of $v$ ever exceeds $\zeta \Delta$. Recall that $\wedge_{v} A 4.2(v)$ implies that the reject degree of each vertex increases by at most $C_{4}-1$.

By Lemma 7.2 , noting that $k_{0}$ was chosen so that $(1-\zeta)^{k_{0}}=\Delta^{-3}$, we have

$$
\operatorname{Pr}(A 4.1(v))<\left(\begin{array}{c}
\Delta^{2} \\
\sqrt{\Delta}
\end{array}\right)(1-\zeta)^{k_{1} \sqrt{\Delta}}<\Delta^{-\sqrt{\Delta}} .
$$

Furthermore, by Lemma $7.3, \operatorname{Pr}(A 4.2(v))<\Delta^{-\sqrt{\Delta}}$.

It is straightforward to verify that each event is independent of all but at most $2 \Delta^{4 k_{1}}$ other events, and the lemma follows by the Local Lemma.

\subsection{Step 2: Finishing Off the Stragglers.}

For each uncoloured vertex $v$, we will select a subset Candidate $v$ of the colours not yet appearing in $N(v)$, whose elements we refer to as candidates. We require the following condition:

(Q4.2) For each uncoloured $v$, there is some $c \in$ Candidate $_{v}$, such that

1. $c$ is not a candiate for any neighbour of $v$, and

2. there is no uncoloured vertex $v^{\prime}$, candidate $c^{\prime}$ of $v^{\prime}$ and $u \in N(v) \cap N\left(v^{\prime}\right)$, with the edge $(u, v)$ coloured $c$ and the edge $\left(u, v^{\prime}\right)$ coloured $c^{\prime}$. 
We then assign each vertex $v$ the colour referred to in (Q4.2). (Q4.2a) ensures that we will then have a proper vertex colouring of $G$, and (Q4.2b) ensures that the maximum reject degree will be at most $C_{1}+C_{2}+C_{3}+C_{4}<$ $C-3$. It only remains that we can select a suitable set of candidates for each uncoloured vertex.

Lemma 7.6 We can choose the sets Candidate such that (Q4.2) holds.

Proof Consider any uncoloured vertex $v$. By property (P3.1), there are at least $\frac{\epsilon \gamma^{3}}{8} \Delta$ colours which do not appear on $N(v)$. To choose Candidate $v$, we simply select 20 of these colours at random. Define $A 4.3(v)$ to be the event that $(\mathrm{Q} 4.2)$ does not hold for $v$. By property $(\mathrm{Q} 4.1), \operatorname{Pr}(A 4.3(v)) \leq$ $\left(40 \sqrt{\Delta} / \frac{\epsilon \gamma^{3}}{8} \Delta\right)^{20}<\Delta^{-9}$. Furthermore, each event is independent of all but at most $\Delta^{4}$ others. Therefore, by the Local Lemma, $\operatorname{Pr}\left(\wedge_{v} A 4.3(v)\right)>0$.

\section{The Aftermath}

And finally, we recolour the reject edges to get our total colouring of $G$.

Proof of Theorem 1.1:

Start with any $\Delta+1$ proper edge-colouring of $G$. Find a $\Delta+1$ vertex colouring such that the reject degree of any vertex is at most $C-3$, as guaranteed in Sections $3-7$. The reject graph will have maximum degree at most $C-2$ and so by Vizing's Theorem, we can recolour the reject edges with $C-1$ new colours, thus yielding a total colouring with at most $\Delta+C$ colours.

\section{Acknowledgements}

We would like to thank Colin McDiarmid for some fruitful discussions. 


\section{Index}

$A_{i}^{\prime} 16$

artificial 11

$\mathrm{Big}_{i} 11$

$C_{1}, C_{2}, C_{3}, C_{4} 15$

$\mathcal{C}_{i} 8$

$\mathcal{C}_{i}^{\prime} 16$

Candidate $w 36,45$

candidate 36,45

colour class 8

coloured 41

colourless 41

Crit $(v) 41$

critical 26, 41

dense 6

dense set 6

$\epsilon 6$

external 7

forbidden colour 26

$\gamma 16$

H 7

$H^{\prime} 12$

internal 7

$K_{i} 9$

kernel 9

Moderate $_{i} 11$

ornery set 9
$\mathrm{Out}_{v} 7$

Overused $_{i} 10$

$P R(v) 17$

reject edge 14

reject degree 14

reject graph 14

$S_{i} 7$

sparse 6

strongly non-adjacent 13

Swappable $_{v} 34$

swapping class 34

Temp $_{i} 16$

$\operatorname{Temp}_{i}(a) 16$

temporarily coloured 16,27

truly coloured 16

$\zeta 42$ 


\section{References}

[1] N. Alon, Restricted colourings of graphs, Surveys in Combinatorics, Proc. 14th British Combinatorial Conference, Cambridge University Press, Cambridge, 1993, 1-33.

[2] N. Alon and J. Spencer, The Probabilistic Method. Wiley (1992).

[3] K. Azuma, Weighted Sums of Certain Dependent Random Variables. Tokuku Math. Journal 19 (1967), 357 - 367.

[4] J. Beck, An algorithmic approach to the Lovász Local Lemma, Random Structures and Algorithms, 2 (1991), 343 - 365.

[5] M. Behzad, Graphs and Their Chromatic Numbers, Ph.D. thesis, Michigan State University (1965).

[6] R. Brualdi in: Problèmes combinatoires et théorie des graphes, Proc. Coll. Intern. C.N.R.S., Paris-Orsay, 1976; J.-C. Bermond, J.-C. Fournier, M. Las Vergnas, D. Sotteau, eds., Editions du CNRS, Paris 1978, page 437.

[7] V. Chavátal, Linear Programming, Freeman (1983).

[8] P. Erdös and L. Lovász, Problems and results on 3-chromatic hypergraphs and some related questions, in: "Infinite and Finite Sets" (A. Hajnal et. al. Eds), Colloq. Math. Soc. J. Bolyai 11, North Holland, Amsterdam, $1975,609-627$.

[9] W. Feller, An Introduction to Probability Theory and its Applications, Vol 1, Wiley (1966).

[10] R. Häggkvist and A. Chetwynd, Some upper bounds on the total and list chromatic numbers of multigraphs, J. Graph Th. 16 (1992), 503 - 516.

[11] H. Hind, An improved bound for the total chromatic number of a graph, Graphs and Combinatorics 6 pp. 153 - 159 (1990).

[12] H. Hind, M. Molloy and B. Reed, Colouring a graph frugally, Combinatorica (to appear). 
[13] H. Hind, M. Molloy and B. Reed, Total colouring with $\Delta+p o l y(\log \Delta)$ colours, SIAM J. Comp. (to appear).

[14] T. Jensen and B. Toft, Graph Colouring Problems, Wiley (1995).

[15] K. Kilakos and B. Reed, Fractionally colouring total graphs, Combinatorica 13, pp. 435 - 440 (1993).

[16] C. McDiarmid. On the Method of Bounded Differences. Surveys in Combinatorics, Proceedings of the Twelfth British Combinatorial Conference (1989), $148-188$.

[17] L. Lovász and M. Plummer, Matching Theory, Annals of Discrete Math. 29, North Holland, Amsterdam (1986).

[18] M. Molloy and B. Reed, A bound on the strong chromatic index of a graph, Journal of Combinatorial Theory (B) 69 (1997), 103 - 109.

[19] M. Molloy and B. Reed, Further algorithmic aspects of the Local Lemma, Proceedings of STOC 1998.

[20] M. Molloy and B. Reed, Graph Colouring with the Probabilistic Method, a book in preparation.

[21] B. Reed, $\chi, \Delta$, and $\omega$, J. Graph Theory (to appear).

[22] V. Vizing, Some unsolved problems in graph theory, Russian Math Surveys 23, pp. 125 - 141 (1968).

[23] H.P. Yap, Total Colourings of Graphs, Lecture Notes in Mathematics 1623, Springer, New York (1996). 